Chapter 17

\title{
Thin-Film Process Technology for Ferroelectric Application
}

\author{
Koukou Suu \\ Additional information is available at the end of the chapter \\ http://dx.doi.org/10.5772/54360
}

\section{Introduction}

Recently thin-film ferroelectrics such as $\mathrm{Pb}(\mathrm{Zr}, \mathrm{Ti}) \mathrm{O}_{3}(\mathrm{PZT})$ and $(\mathrm{Ba}, \mathrm{Sr}) \mathrm{TiO}_{3}$ (BST) have been utilized to form advanced semiconductor and electronic devices including Ferroelectric Random Access memory(FeRAM), actuators composing gyro meters, portable camera modules, and tunable devices for smart phone applications and so on. Processing technology of ferroelectric materials is one of the most important technologies to enable the above- mentioned advanced devices and their productions.

In this paper, we will report our development results of ferroelectric thin film processing technologies including sputtering, MOCVD and plasma etching as well as manufacturing processes for FeRAM, MEMS(actuators, tunable devices) and ultra-high density probe memory.

\section{FeRAM technology}

Ferroelectric Random Access Memory (FeRAM) is a main candidate of next generation nonvolatile random access memory. As its density has been continuously increasing, its applications have spread from RFID card to Nonvolatile latch circuit with low power consumption. FeRAM has a ferroelectric thin films sandwiched by rare metal electrodes and we have established mass production capability on integrated FeRAM solution, consisting of electrode film deposition, PZT thin-film deposition, etching/ashing, anneal and passivation deposition.

\subsection{Mass productive sputtering technology for perovskite oxide thin-film}

We have been developing mass production technology for perovskite oxide thin-film such as PZT for a long period [1-5], since we consider these materials as the most promising can- 
didate for ferroelectric material used in FeRAMs (Ferroelectric Random Access Memories) as a non-volatile memory device, piezoelectric MEMS (Micro Electro Mechanical System) devices due to its longer period of research, existence of actual production, manufacturing capability within the tolerable temperature range of general Si LSI technology.

The sputtering was selected for mass production technology of perovskite oxide thinfilms owing to the following factors: (1) Good compatibility with conventional Si LSI processes. (2) Superb controllability of film quality (e.g. film composition), which enabled relatively easy thin film deposition. (3) Better possibilities of obtaining uniform surfaces in large diameter substrates (e.g. 6-8 inch). (4) Sputtering was plasma processing which was promising for deposition and heat treatment at low temperature. (5) Feasibility of highspeed deposition. (6) Same deposition method as electrodes ( $\mathrm{Pt}, \mathrm{Ir}, \mathrm{Ru}$, etc.), which will facilitate in-situ integration. (7) Present difficulties and lack of future potentialities in other technologies.

With emphasis on both mass production capability and advanced process capacity of perovskite oxide thin-film sputtering, we consider the following factors as important for development:

1. Throughput

Compared to the other processes (e.g. electrode deposition), the deposition speed of perovskite oxide material sputtering was considerably slower and thereby limited the throughput. Though two ways for improving the throughput, high-speed deposition and thinner film deposition, are considered, the former is more promising for the improvement of throughput, while the latter is apt to cause deterioration of film characteristics.

\section{Control of film composition}

Film composition determines other film qualities (crystal structure, electric characteristics). Since volatile elements were included in the perovskite oxide materials, they were sensitive and easily fluctuated according to temperature or plasma status. Film composition control is the fundamental factor in this process.

3. Uniformity over large diameter substrates

Large diameter substrates up to 8 inch were expected to be used for mass production of perovskite oxide thin-film. Film thickness and uniformity of film quality were the keys to mass production.

\section{Process stability / reliability}

Under the circumstances where there were many unknown factors such as new materials, ceramic targets, insulator sputtering, etc., process stability / reliability was the more pertinent factor. In fact, there was a problem in film composition that changed over time.

5. Prevention of particles

While the characteristics, such as ceramic targets or insulating thin film, increased the mechanical factors (e.g. adherence, thermal expansion) for particle occurrence, they were also 
producing electric factors (e.g. dielectric breakdown due to charge-up) and made the measures difficult to obtain.

\subsection{Optimization of sputtering processes for perovskite oxide thin-film}

We adapted the RF magnetron sputtering method for perovskite oxide thin-film sputtering. As for the PZT thin-films, sputtering methods included high-temperature deposition, where film deposition was made at substrate temperatures above $500^{\circ} \mathrm{C}[6,7]$, and low temperature deposition, where the films were deposited at room temperature and then crystallized by post-annealing process. The improvement in high-speed deposition, film composition control, and stability of sputtering processes is described in the following.

If the ceramic target with inferior thermal conductivity is used, application of high power leads to the destruction of the target. By adopting the backing plate with high cooling efficiency and high-density target, higher deposition rate by an increase in sputtering power is achieved.

The film composition control can be translated into the volatile element (e.g. Lead) control within film. Various factors that influence the volatile element within film are thought to exist (Fig. 1) and we have investigated the influence of sputtering conditions, strength of magnetic field and electric potential of substrate.

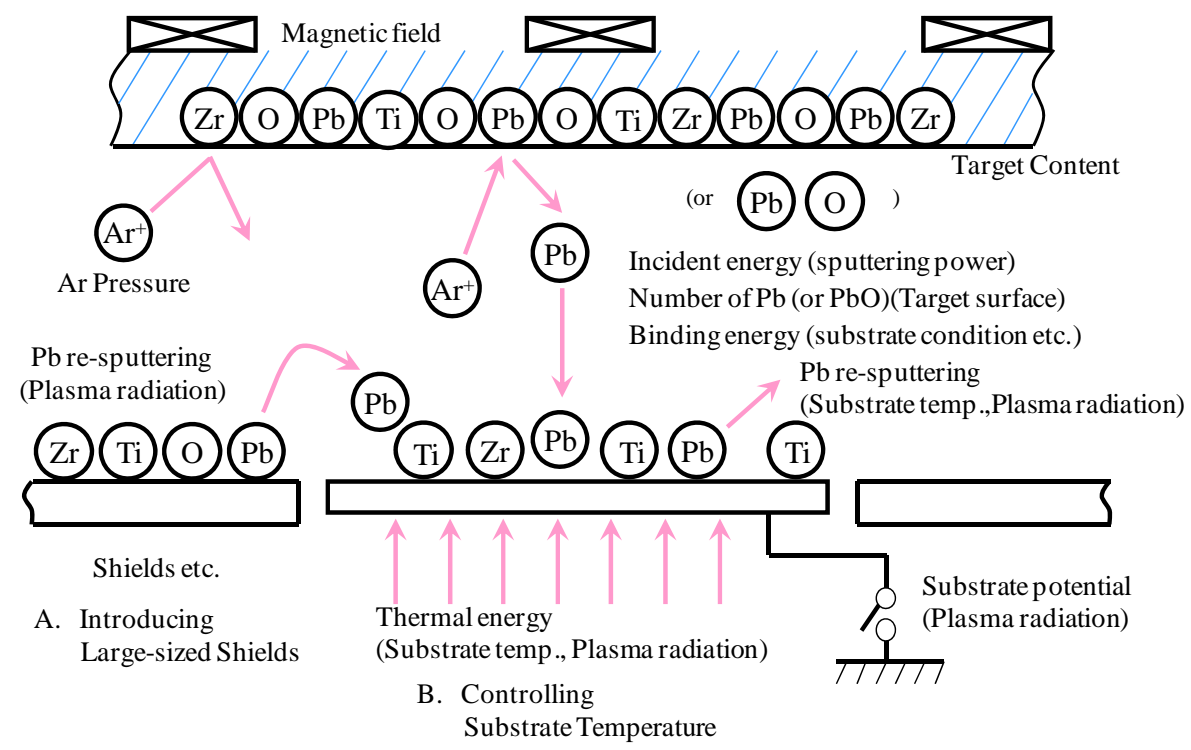

Figure 1. Various factors that influence content with PZT Film. 
In addition to the known characteristics in perovskite oxide thin-film sputtering that volatile elements easily fluctuate, it was confirmed that volatile elements within film were unstable even when the deposition was performed under identical conditions. The conceivable causes for that phenomenon were the instability of the target, fluctuation of temperature in the sputtering chamber, and variation of plasma status over time.

Main problem in perovskite oxide thin-film is the change in the volatile element content with the passage of sputtering time. As for the PZT, continuous sputtering of $2.0 \mathrm{kWh}$ showed approximately a $30 \%$ decline in lead content compared to that at the beginning. It was determined that the reason for this problem was the change in plasma status. As shown in Fig. 2, when insulating PZT film adhered to the shields of the ground potential, charge-up occurred, the impedance of the system changed, plasma was pushed to the center of the chamber, exposure to plasma was enhanced, and as a consequence, $\mathrm{Pb}$ content within film is reduced. In order to stabilize the status of plasma, we installed a stable anode, that is, an anode that avoided charge-up due to the adhesion of insulating PZT film and maintained the role as an anode. Consequently, as can be seen in Fig. 3, stability of $\mathrm{Pb}$ content within film in continuous sputtering has been confirmed.

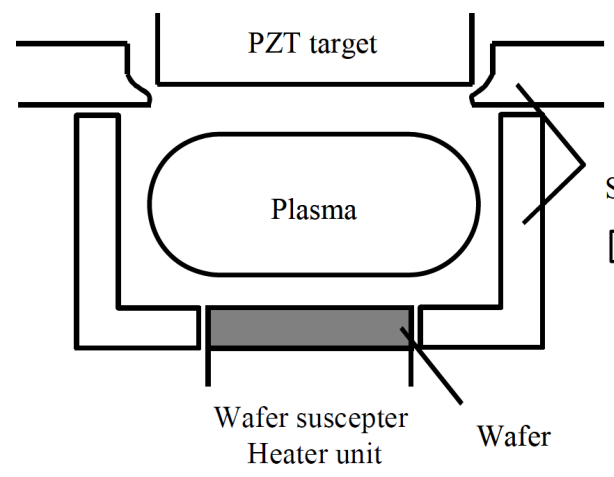

(a) as cleaned state

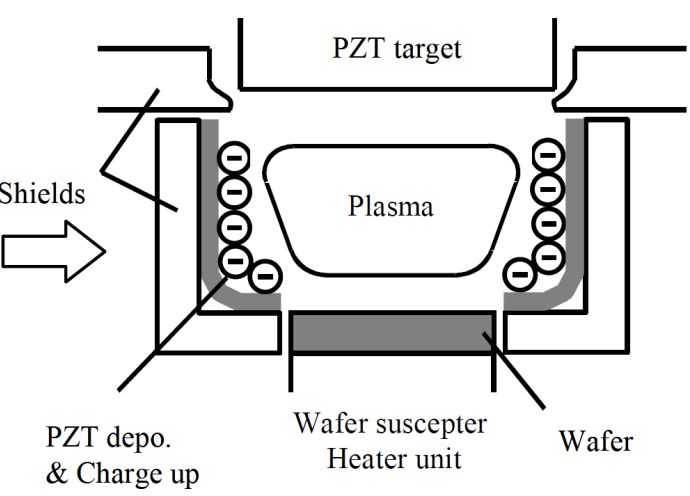

(b) PZT deposited state

Figure 2. Change in plasma status which is responsible for $\mathrm{Pb}$ content variation.

Fig. 4 shows an example of thickness and Pb content uniformity in a PZT film on 8" substrate. Both thickness and $\mathrm{Pb}$ content uniformity varied according to the deposition condition and was minimized around the sputtering pressure of $1.0 \mathrm{~Pa}$. Thickness and $\mathrm{Pb}$ content uniformity represent good result as low as $\pm 1.9 \%$ and $\pm 1.1 \%$, respectively. These uniformities were also confirmed as stable, thereby satisfactorily meeting the requirements of mass production. 


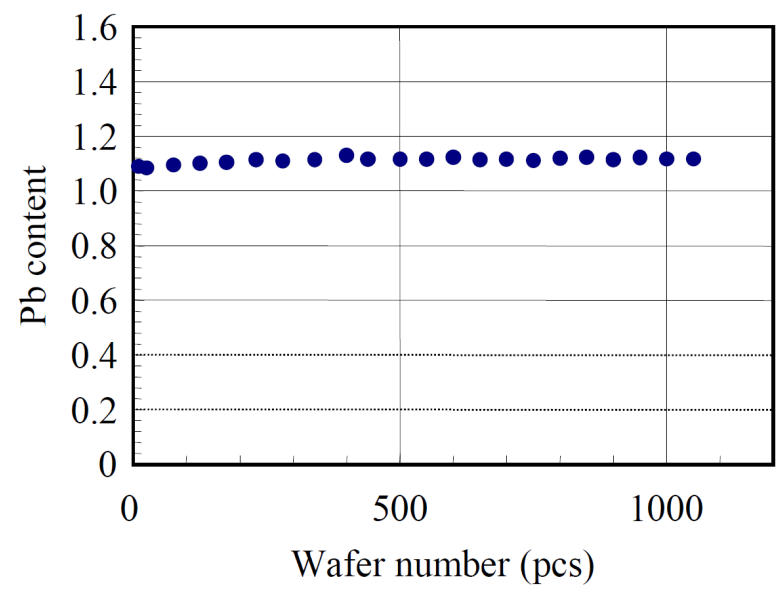

Figure 3. Stable transition of $\mathrm{Pb}$ content within film in continuous sputtering.

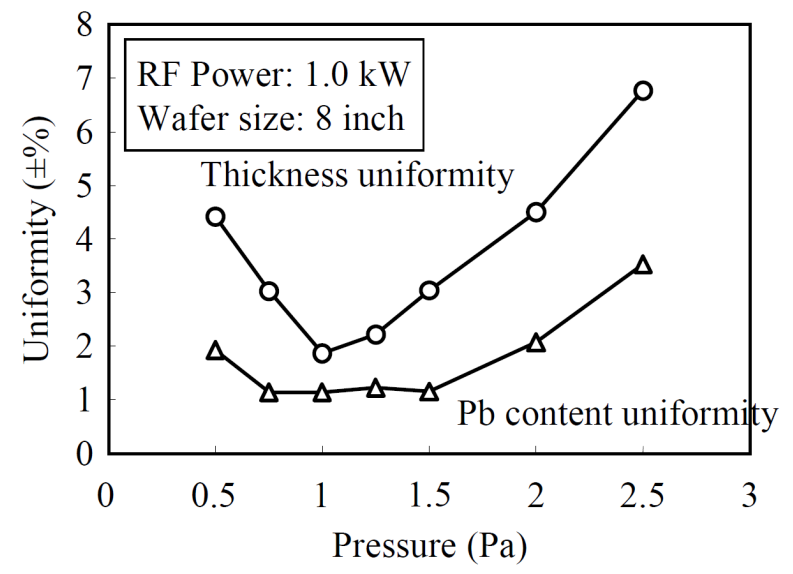

Figure 4. Uniformity in 8 inch area.

\subsection{Sputtered PZT thin-films for non-volatile memory application}

In this section, we introduce one of its achievements, the ferroelectric characteristics of PLZT capacitors for FeRAM. We used the multi chamber type mass production sputtering system equipped with an exclusive sputtering module for ferroelectric materials, CERAUS ZX-1000 from ULVAC. This system has the following features in addition to features such as easy maintenance, short exhausting time, short down time, etc. 
1. Can mount $300 \mathrm{~mm}$ (12 inch) in diameter targets and process large diameter substrates of $200 \mathrm{~mm}$. At present, deposition of PZT thin-films on 6 and 8 inch substrates are performed using 12 inch single ceramic target.

2. Including the heat chamber, this system has five process chambers, thereby achieving high flexibility. The system is presently executing the following in-situ processes as standard: pre-heating of substrate $\rightarrow$ substrate sputtering $($ e.g. $\mathrm{Ti}, \mathrm{TiN}, \mathrm{Pt}) \rightarrow$ ferroelectric material sputtering.

3. As a substrate heating mechanism, this system was capable of precise and rapid heating in a wide range from low to high temperatures, with the aid of an electrostatic chuck type hot plate, in addition to lamp heating.

4. This system used RF sputtering for ferroelectric deposition and counters RF noises.

Fig. 5 shows the transition of switching charge $\left(\mathrm{Q}_{\mathrm{sw}}\right)$ and saturation characteristics of a Pt/ $\mathrm{PZT}(200 \mathrm{~nm}) / \mathrm{Pt}$ capacitor measured at $5 \mathrm{~V}$. $\mathrm{Q}_{\mathrm{SW}}$ with $5 \mathrm{~V}$ applied was approximately 34 $\mu \mathrm{C} / \mathrm{cm}^{2}$ and saturation voltage of $90 \%\left(\mathrm{~V}_{90 \%}\right)$ was 3.1-3.2 V. The composition of PLZT film, which contained added $\mathrm{Ca}$ and $\mathrm{Sr}$ for the improvement of retention and imprint characteristics, was excellent.

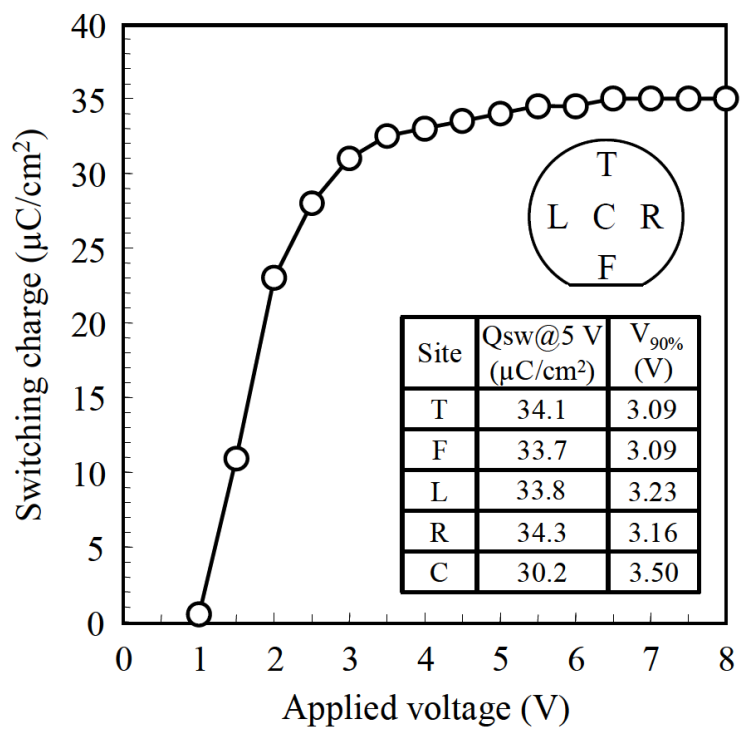

Figure 5. Transition of switching charge of Pt/PZT(200 nm)/Pt capacitor.

Fig. 6 shows the stability of $\mathrm{Q}_{\mathrm{sw}}$ in continuous sputtering of 1000 substrates. The result showed high stability sufficient for mass production. Also, reference data was the data before chamber cleaning. The fact that it was equivalent to the data after chamber cleaning demonstrates the reliability of the system process. 


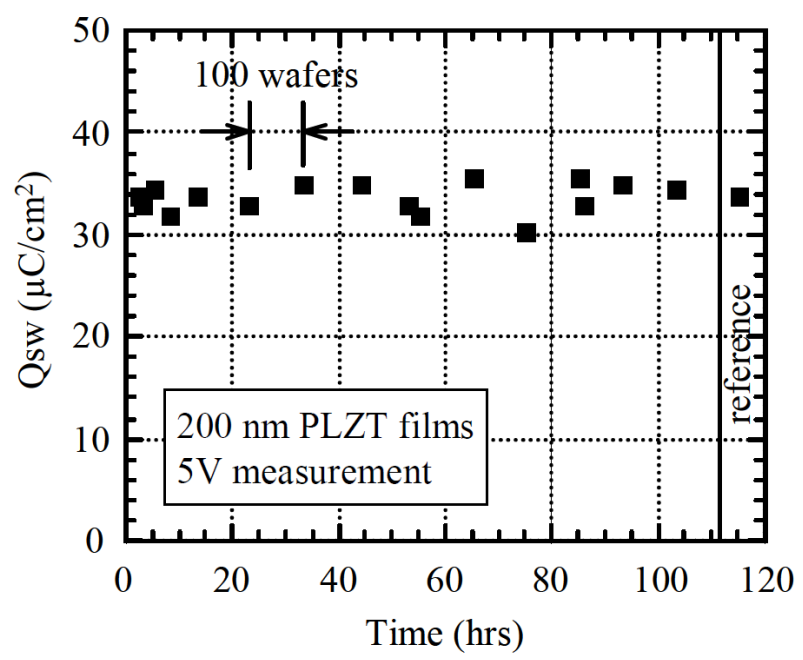

Figure 6. Stability of Qsw in continuous sputtering of 1000 substrates.

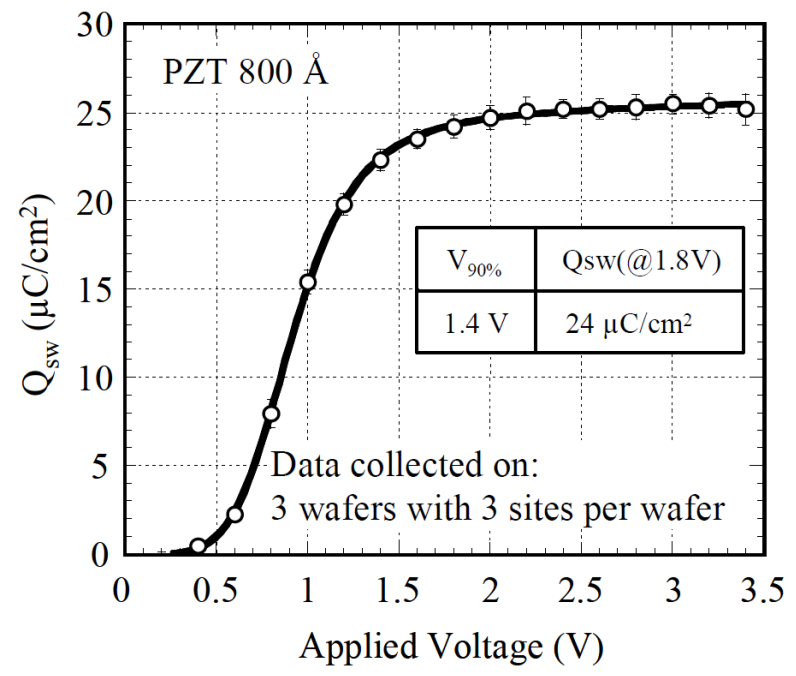

Figure 7. Transition of switching charge of IrOx/PZT(80 nm)/Pt capacitor.

Further improvement of ferroelectric performancs is needed because scaled thinner capacitor (sub $100 \mathrm{~nm}$ ) is demanded for next generation. Pb deficient surface layer is confirmed to be responsible for degradation of ferroelectric performance. Bottom electrode and PZT deposition process were modified to further improve the ferroelectric performance and achieve 
thinner capacitor with good performances. [8]. Fig. 7 shows the $\mathrm{Q}_{\mathrm{sw}}$ transition curve of a Ir$\mathrm{Ox} / \mathrm{PZT}(80 \mathrm{~nm}) / \mathrm{Pt}$ capacitor measured at $3 \mathrm{~V}$. $\mathrm{Q}_{\mathrm{sw}}$ with $1.8 \mathrm{~V}$ applied was approximately 24 $\mu \mathrm{C} / \mathrm{cm}^{2}$ and $\mathrm{V}_{90 \%}$ was $1.4 \mathrm{~V}$. Fig. 8 also shows the fatigue characteristics of voltage application at $2 \mathrm{~V}$. $\mathrm{Q}_{\mathrm{SW}}$ was not decreased even after switching in $10^{9}$ cycles.

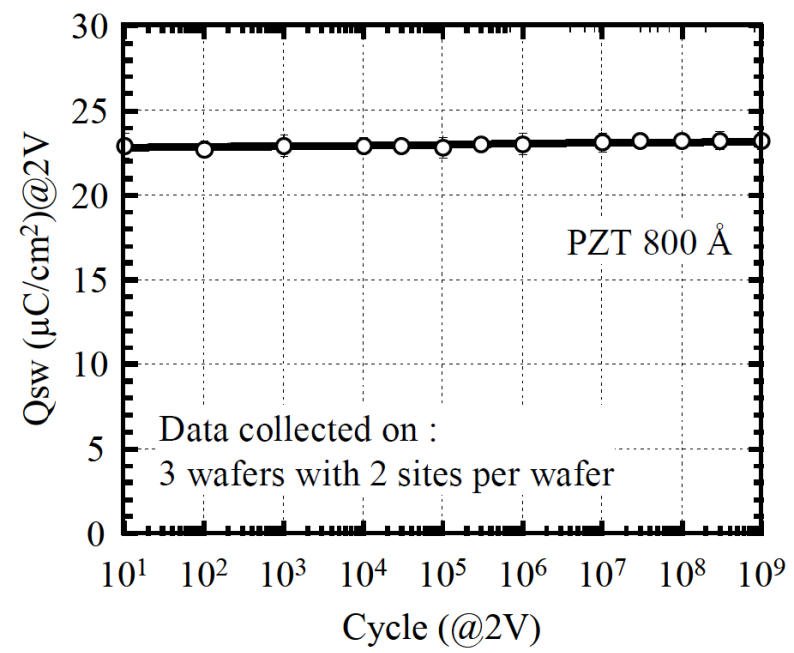

Figure 8. Fatigue characteristics of IrOx/PZT(80 nm)/Pt capacitor.

From above results, sputter-derived PZT capacitor was proved to be suitable for $0.18 \mu \mathrm{m}$ technology node.

\subsection{MOCVD technology for non-volatile memory application}

For next-generation FeRAMs beyond $0.18 \mu \mathrm{m}$, because it is necessary to achieve further larger packing densities and integration with logic devices, thinner films of ferroelectric associated with shrinking of the thickness of ferroelectric capacitors and the three-dimensional structures associated with shrinking of the capacitor areas are demanded. In addition, it is necessary to achieve thinner films of ferroelectric in parallel with its higher quality in order to meet the demand of still lower voltage drives for device performance. It is said that MOCVD (Metal organic chemical vapor deposition) technology can meet these demands. While depositions are physically made by plasma collision in sputtering processes, depositions are made on heated substrates by chemical reaction among source gases in MOCVD. Therefore, denser crystalline films are easily obtainable, and it is possible to achieve thinner films and higher quality. In addition, uniform deposition can be obtained also on three-dimensional structure, and it is considered that good step coverage can be obtained. Because of these features, MOCVD is the prime candidate of deposition technologies for next-generation FeRAMs in mass production, and its development is making progress. 
First, the features of MOCVD are briefly explained. In the method of MOCVD, raw material that was changed into organic metal with high vapor pressure is led to a substrate, and is thermally reacted with a reactant gas (such as oxygen) on the substrate to form a deposition. Methods for the gasification of organic metals are classified into two groups. One of them is the sublimation of solid raw materials (sublimation method). After a certain vapor pressure is obtained by heating a solid raw material using a heater, it is transported with carrier gas. In the second method, a liquid raw material or solid raw material is melted in an organic solvent. Then, after the solution is vaporized, it is transported with carrier gas (vaporization method). In the case of the former, the deposition rate is small. In the case of the latter, there is a problem of the instability of vaporization. For the target of mass-production system development, we adopted the latter vaporization method from the beginning of the development. The problems that need to be resolved are as follows (see Fig. 9):

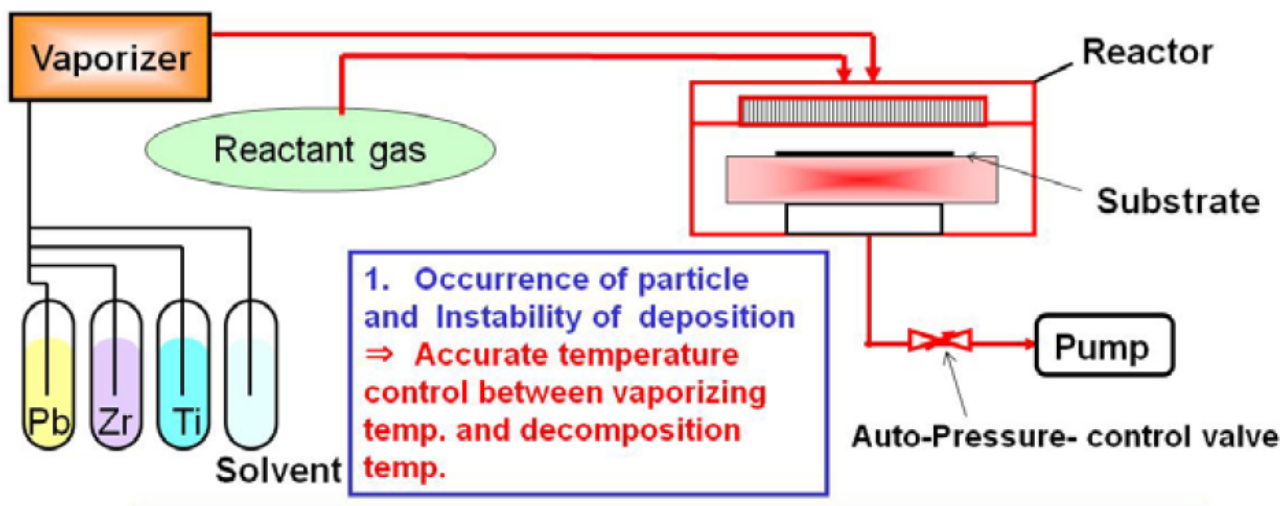

2. Difficulty of mixing precursor gas with oxygen

3. Uniformity on large substrate

$\Rightarrow$ (1) Chamber configuration optimized for controlling uniform gas

flow by simulations and experiments

(2) Substrate heater system heating wafer uniformly

Figure 9. Features of MOCVD Module

1. Vaporization and transport of multi-element raw materials In MOCVD using the vaporization of a ferroelectric solution, because a multi-element organic metal material is vaporized and transported, stable vaporization is difficult, and there is a possibility that precipitation or decomposition may occur in a pipe during transport.

2. Mixture of gases whose molecular weights are mutually different It is difficult to uniformly mix a reactant gas such as oxygen and an organic raw material whose molecular weights are vastly different.

3. Uniformity of large-diameter substrates For the film thickness and the composition of a multi-element oxide thin film PZT, in-plane uniformity in 8 inches is required. 
4. PTZ film properties Because of high-temperature processes and in-situ crystallization in the processes, the correlation between the kind of a raw material and a process is sensitive, and the control of a film composition and crystalline orientation is difficult. Therefore, necessary performance of films is difficult to obtain.

In order to establish MOCVD mass-production technology, it is absolutely necessary to resolve the above problems in parallel, and advanced vaporization technology, mixing technology, and reaction control technology are simultaneously required. We combined ferroelectric deposition technology for FeRAMs, module design technology, and CVD equipment technology, which were provided to users until now, and completed full-fledged MOCVD equipment for mass production. The major features include the following four points:

1. The reproducibility in the continuous operation of vaporization and the gas transport to a substrate that controls condensation and decomposition were achieved by accurate temperature control for each part of equipment and the optimization of vaporization conditions. Consequently, film thicknesses within $\pm 2 \%$ and the reproducibility of the PZT composition were kept with no mechanical maintenance, and a running test for 1,000 substrates was successful as shown in Fig. 10. [9]

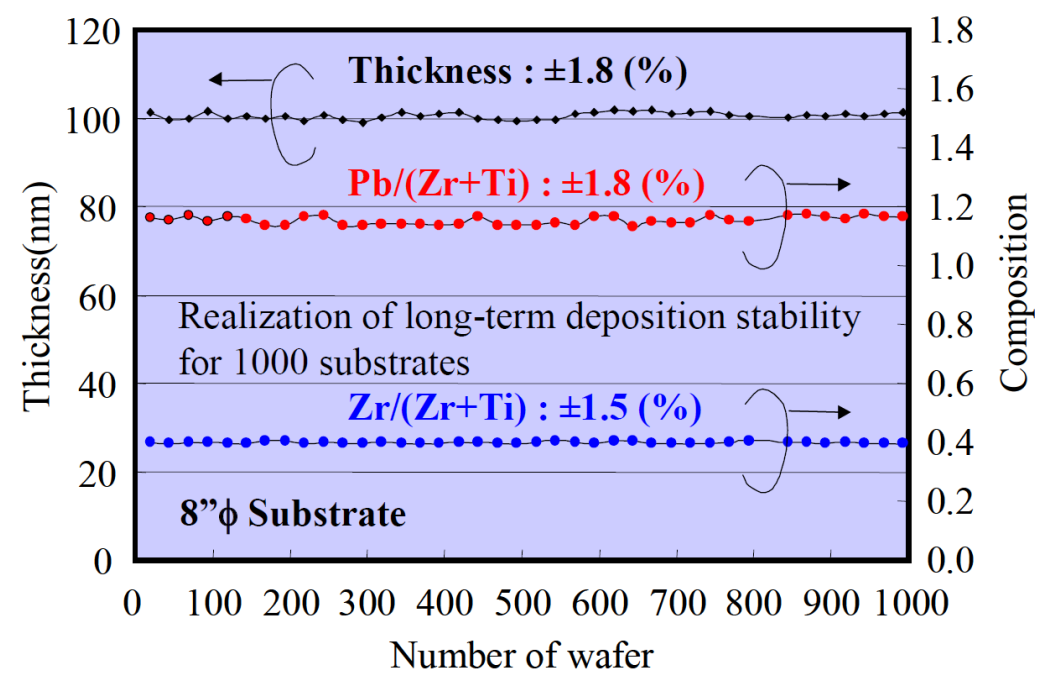

Figure 10. Reproducibility in MOCVD system (film thickness and PZT composition)

2. Because the design of the equipment was conducted in consideration of the flow of gases and the mixture between a raw material gas and a reactant gas on the basis of simulations, film thickness distribution within $\pm 3 \%$ on 8 inch substrates was achieved as shown in Fig. 11. 


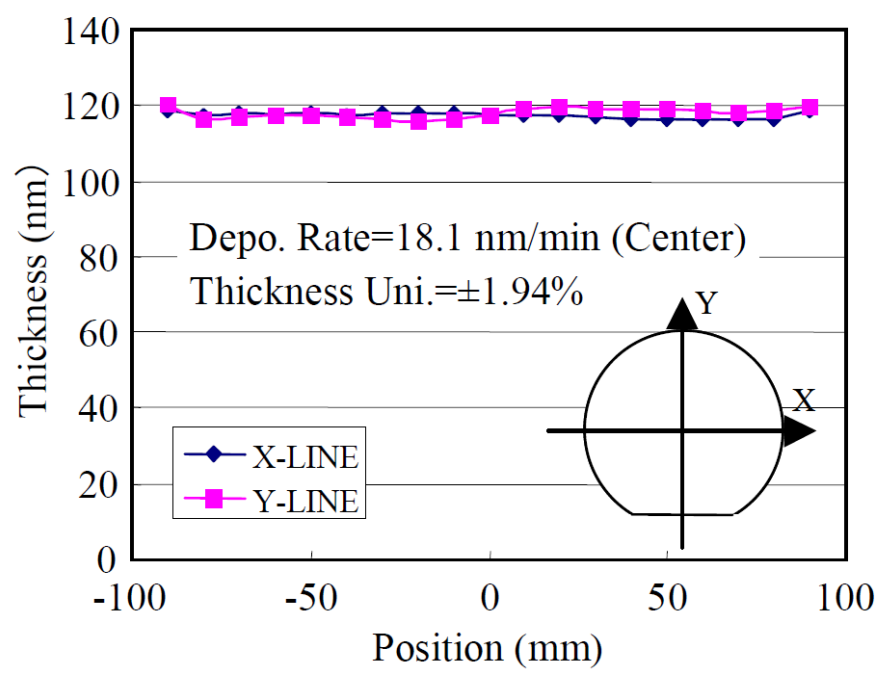

Figure 11. In-plane uniformity of PZT film thickness

3. Because of the development of a new heater, including the optimization of the shape of the heater, the temperature distribution of 8 inch substrates can be continually controlled within $\pm 3^{\circ} \mathrm{C}$. Consequently, in-plane uniformity within $\pm 3 \%$ was achieved for both film thicknesses and the composition as shown in Fig. 12. [10]

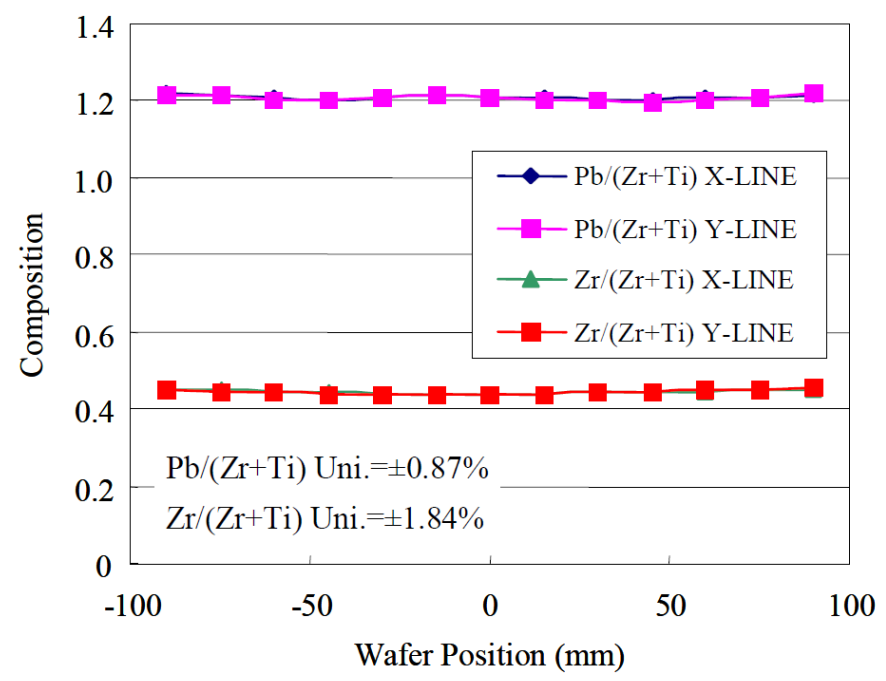

Figure 12. In-plane uniformity of PZT composition 
4. By taking advantage of the progress of the equipment hardware as described above, the optimization of processes was conducted, and PZT thin films can be controlled to ensure preferential orientation in the $<111>$ direction as shown in Fig. 13. Consequently, the formation of PZT films within $100 \mathrm{~nm}$ that have capacitor properties with a $1.5 \mathrm{~V}$ low voltage drive is achieved as shown in Fig. 14. [11,12] In addition excellent endurance properties which are over $10^{10}$ cycles were obtained for $73 \mathrm{~nm}$-PZT in Fig 15.

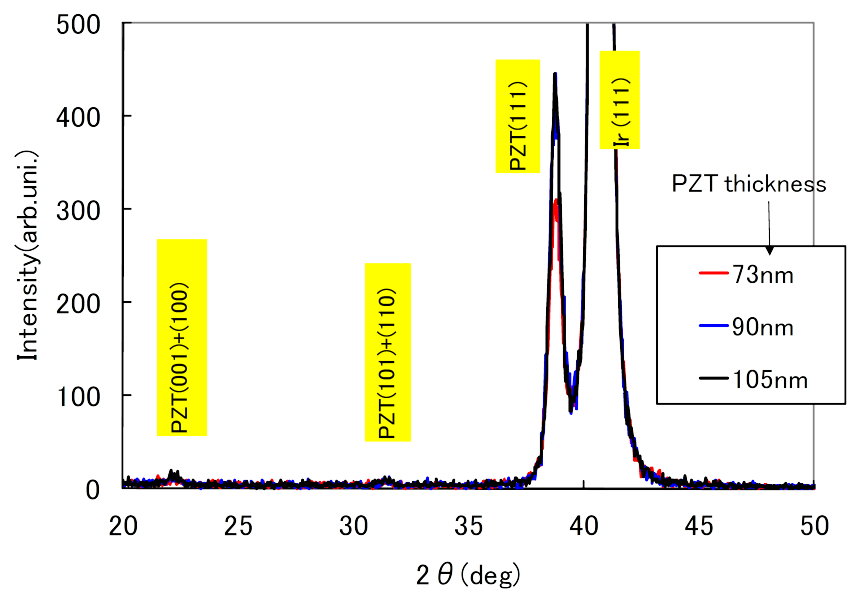

Figure 13. XRD spectrum for various thickness PZT

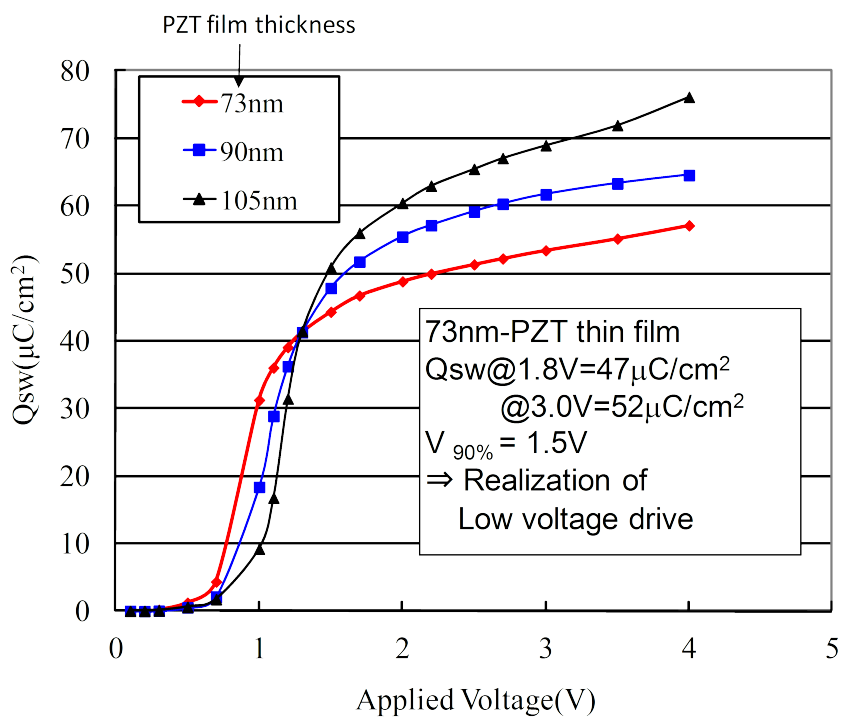

Figure 14. Characteristics of MOCVD-PZT film (applied voltage dependence of switching charge) 


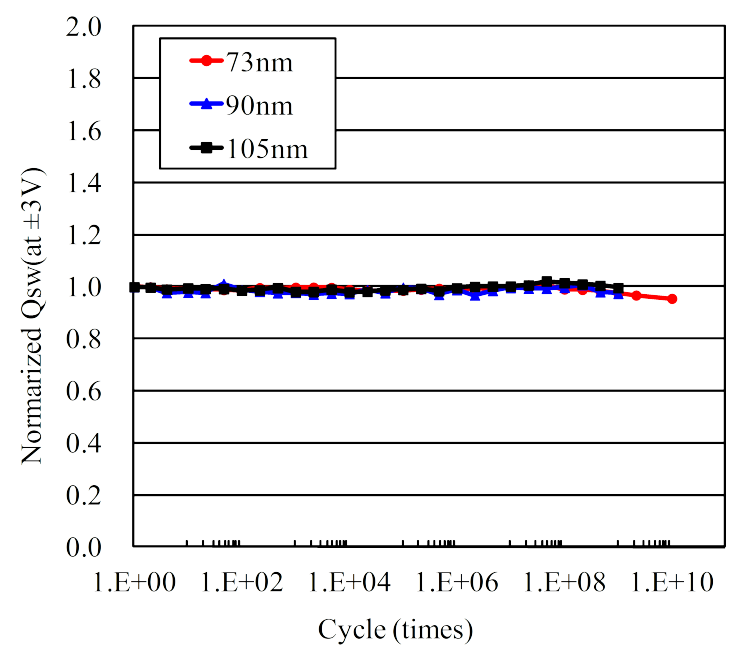

Figure 15. Endurance properties of MOCVD-PZT films

Recently mass production tool for $300 \mathrm{~mm}$ Si wafer was developed and excellent in-plane uniformity less than $1.5 \%$ for thickness and PZT composition were obtained.

\subsection{Etching technology}

\subsubsection{Issues of ferroelectric etching technology}

Conventionally, the piezoelectric elements have been fabricated by chemical wet etching [13] or argon ion milling. With the miniaturization of MEMS, there have been increasing demands for dry etching with the excellent shape controllability as semiconductor technology. Recently dry etching technique for MEMS using PZT was reported. [14,15] The Pt, Ir and other rare metal electrodes and the PZT ferroelectric thin films that compose piezoelectric elements react poorly with halogen gases and their halides have low vapor pressures. For these reasons, these materials are called hard-to-etch materials. The following technical issues are important for dry etching of the PZT ferroelectric thin films for not only FeRAM but also MEMS productions:

1. Etching selectivity to resist mask and the bottom rare metal electrode

A piezoelectric element film consists of PZT with a thickness of several micrometers and the rare metal electrodes with a thickness of about $100 \mathrm{~nm}$. Generally, the bottom electrode is left after the PZT etching. Therefore, a low etching rate for the bottom electrode, the socalled high etching selectivity, is important as a PZT etching condition.

2. Adhesion of conductive deposit to the pattern sidewalls and damage to PZT

The materials are hard to etch, and their etching products easily adhere to the pattern sidewalls, and result in leaks between the top and bottom electrodes. What is worse, the pattern 
sidewalls are exposed to reactive gas plasma during etching, and tend to suffer lead and oxygen coming out and other damages.

3. Plasma stability during continuous processing

Adhesion of etching products to chamber walls, especially the RF introduction window that generates plasma, causes instabilities of plasma and deteriorates the etching rate and the shape reproducibility. Avoiding of adhesion of etching products to chamber walls is important for mass production.

4. Uniformity of etching rate within wafer

As in the case of (1), to stop the thick PZT at the thin bottom electrode after etching, the uniformity of etching rate within wafer is important.

\subsubsection{Ferroelectric etching systems and process for mass production}

As the piezoelectric PZT etching systems, this section explains about Apios NE series made by ULVAC, Inc. The etching module is equipped with the ISM (Inductively Super Magnetron) plasma source that can generate low-pressure and high-density plasma. Fig. 16 shows a drawing of the etching module. Table I shows the comparison between the normal ICP type plasma source and the ISM plasma source. The RF antenna is mounted in the upper part of the etching chamber, so that RF is introduced through the quartz window into the etching chamber to generate plasma. The uniformity of the etching rate within wafer can be easily optimized by positioning permanent magnets under the antenna. Fig. 17 shows the uniformity of PZT etching rate within 6 inch wafer. A high uniformity $(<+/-5 \%)$ was realized by means of optimization to permanent magnet layout. A STAR electrode is provided between the antenna and the quartz window to control adhesion of etching products to the quartz window by applying RF to the STAR electrode. The substrate is held on the electrostatic chuck. The substrate temperature is controlled by introducing Helium to the backside of the substrate. The ion energy is controlled by applying RF power to the substrate. The materials to be etched are non-volatile, and the etching products adhere to the shield located in the chamber. The temperature of the shield is kept constant by heater, so process is high stability.

\begin{tabular}{ccc}
\hline & ISM & ICP \\
\hline Plasma density $\left(\mathrm{cm}^{-3}\right)$ & $1 \times 10^{10} \sim 1 \times 10^{11}$ & $5 \times 10^{9} \sim 5 \times 10^{10}$ \\
\hline Operating pressure $(\mathrm{Pa})$ & $0.07<\mathrm{P}<7$ & $0.5<\mathrm{P}<50$ \\
\hline Uniformity & Optimized magnetic layout & Determined by chamber structure \\
\hline Damage & Plasma density and substrate bias can be & \\
\hline Repeatability, stability & controlled independently. & High pressure process causes re- \\
& etching $->$ better repeatability & deposition. \\
\hline Maintenance & Chamber structure is simple for easy & maintenance.
\end{tabular}

Table 1. Inductively super magnetron (ISM) plasma performances. 


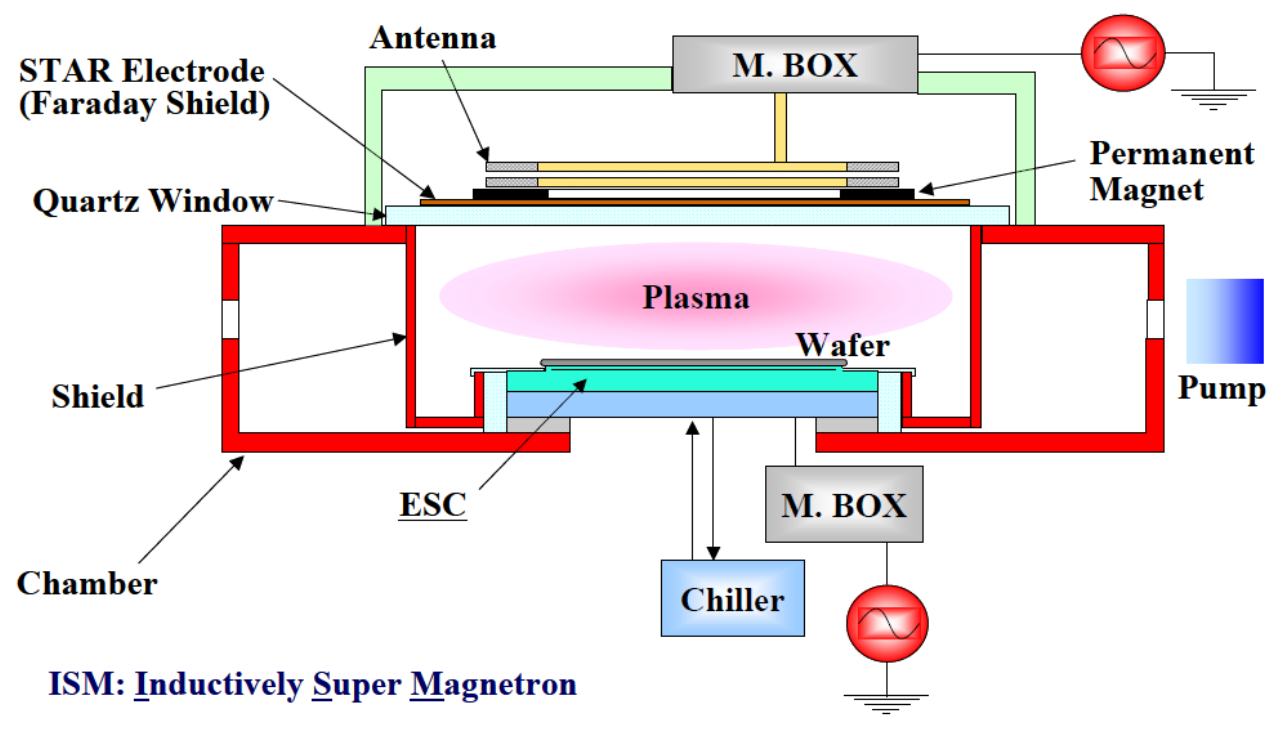

Figure 16. Etching chamber with inductively super magnetron (ISM) plasma performances

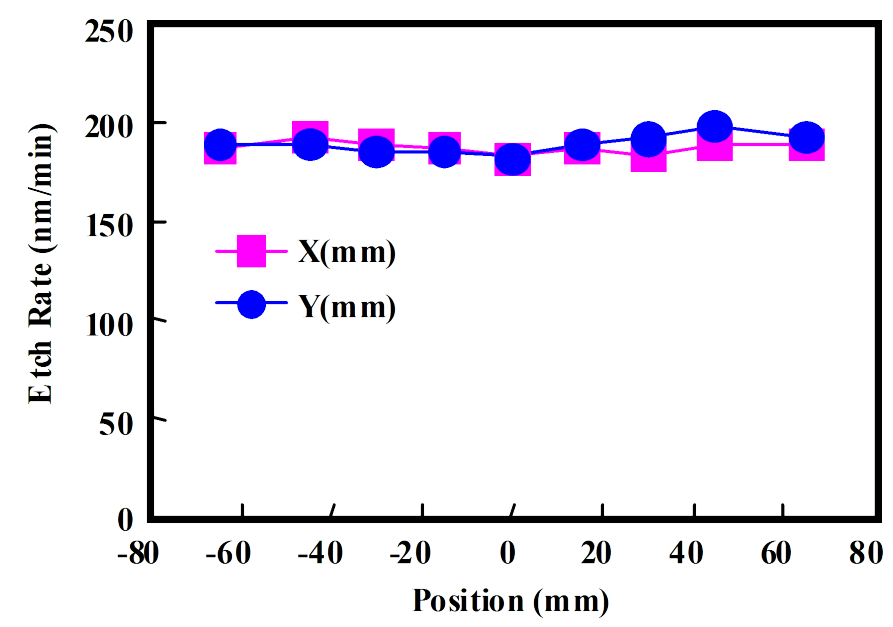

Figure 17. PZT etching uniformity

\subsubsection{Mass productive high temperature etching technology for high dense FeRAM}

Dry etching techniques are used for the patterning of FeRAM device. It is difficult to etch the material of FeRAM such as the noble metal and PZT, because these have low reactivity 
with halogen gas plasma and these halides have low vapor pressure. The photo-resist is used as etching mask for the patterning of FeRAM memory cell. But the etching selectivity to photo-resist is low. Therefore the etching profile becomes low taper angle. Since FeRAM device shrinks down recently(0.35- $\mu$ m-design rule or lower $)$, high temperature etching was developed for high density FeRAM device. Furthermore, FeRAM device changed to the stack structure from planer structure. At that time, the top electrode, PZT and the bottom electrode are etched in a series by using of hard-mask (Fig. 18). [16]

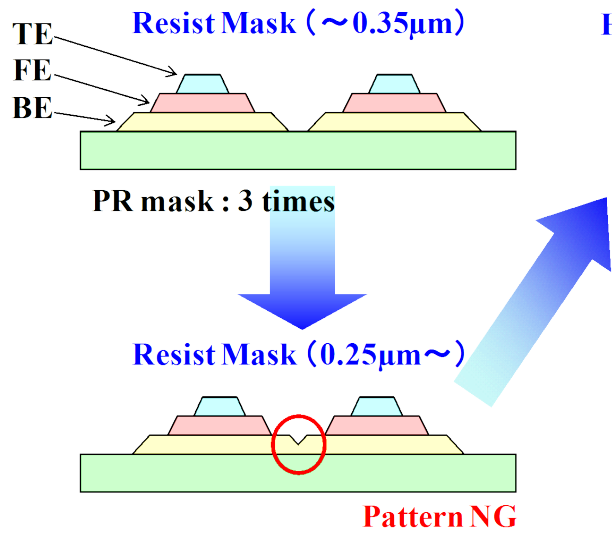

Pattern NG

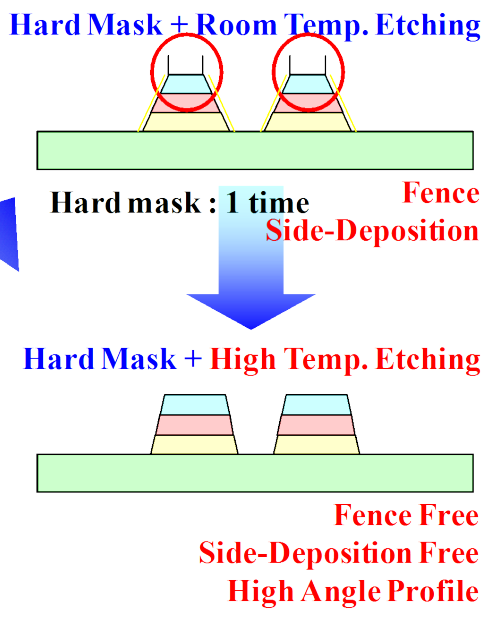

Figure 18. Necessity of high-temperature etch

This section explains high temperature etching system ULHITE series made by ULVAC, Inc. This etching module is equipped with ISM plasma source. Most important feature is the novel electro-static-chuck (ESC) type hot plate stage at a temperature up to $450^{\circ} \mathrm{C}$, and this stage can be supplied high bias power. The process chamber, variable conductance valve, pumps and gas exhaust are heated up. The deposition shields equipped in process chamber are also heated up to $200^{\circ} \mathrm{C}$. The effect is reducing the deposition during the etch process, and high process stability is achieved.

Fig. 19 shows repeatability of Ir etching time for 300-wafers running test. The plasma cleaning were carried out in every 25-wafers. Excellent stability was confirmed. For next generation FeRAM devices, the continuous etching among the top electrode, ferroelectrics and bottom electrode by use of one mask is need. And the high taper angle and no sidewall deposition are needed for etching process.

Fig. 20 shows the results of FeRAM capacitor etching of stack structure dependence of the stage temperature. The halogen gases were used in etching process. Thus the higher profile can be obtained by higher stage temperature. Fig. 21 shows repeatability of etching profile for 300-wafers running test. Excellent repeatability of etching profile was confirmed. 


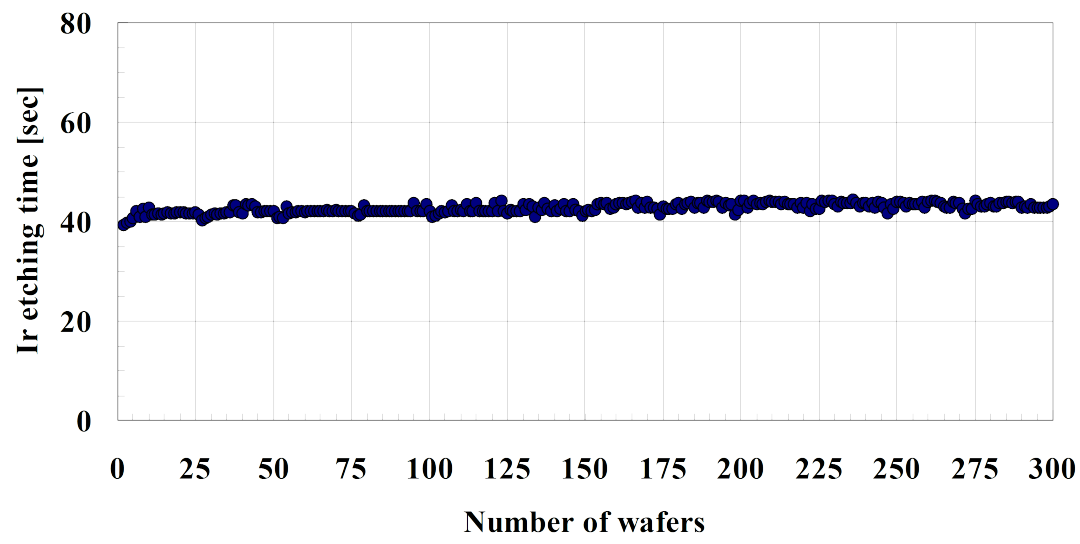

Figure 19. Repeatability of Ir etching time for 300-wafers running test

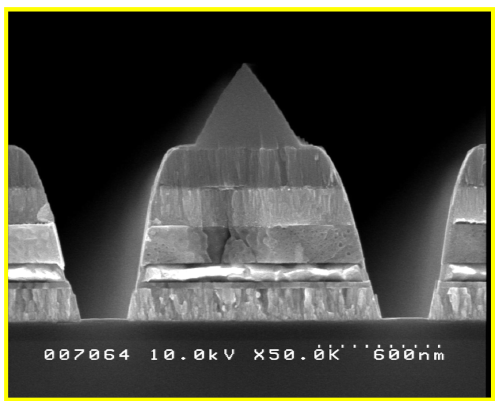

(a) Taper angle $=74$ degree

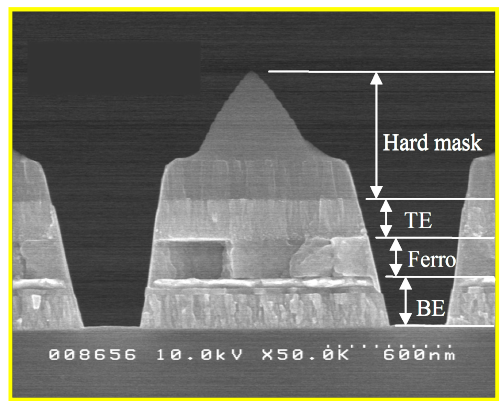

(b) Taper angle $=80$ degree

Figure 20. Stage temperature dependency of taper angle ( (a) $300^{\circ} \mathrm{C}$, (b) $400^{\circ} \mathrm{C}$ )

Usually, high density device is required for non-volatile memory. Therefore the high temperature etching technique contributes to realize the next generation FeRAM devices production.

\section{Technology for MEMS application}

\subsection{Sputtered PZT piezoelectric films for MEMS application}

A multi-chamber type mass production sputtering systems for electronic devices SME-200 equipped with an exclusive sputtering module described above. PZT films have been deposited on 6 inch diameter silicon substrates. 


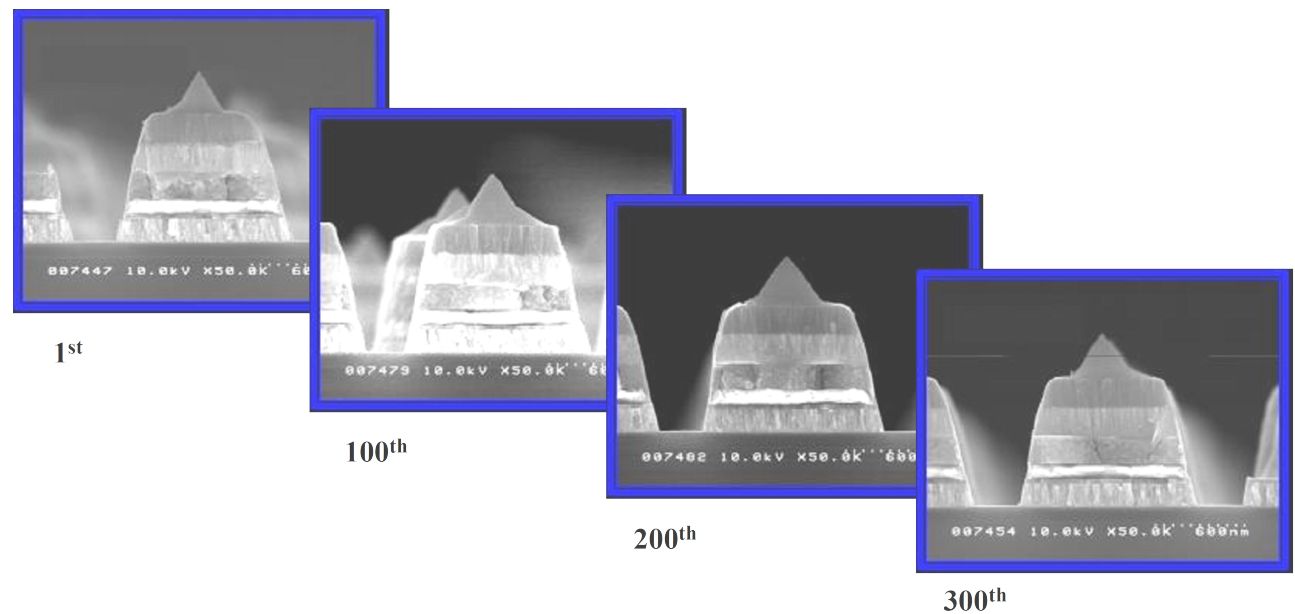

Figure 21. Repeatability of etching profile for 300-wafers running test

The platinum bottom electrodes, whose orientation is (111), were deposited on the substrate. The PZT films were deposited under $\mathrm{Ar} / \mathrm{O}_{2}$ mixed gas atmosphere of $0.5 \mathrm{~Pa}$. Substrate temperature was heated up to around $550^{\circ} \mathrm{C}$. After the deposition, PZT films were conducted with no thermal treatments such as post-annealing. PZT films were deposited with relatively high growth rate about $2.1 \mu \mathrm{m} / \mathrm{h}$ and these thicknesses were from 0.5 to $3.0 \mu \mathrm{m}$ in consideration of piezoelectric MEMS applications. The ceramic target with $\mathrm{Zr} / \mathrm{Ti}$ ratio of 52/48, in which $30 \mathrm{~mol}$ $\%$ excess $\mathrm{PbO}$ was added for the compensation of the lead re-evaporation from the films, was used in order to obtain PZT films near the stoichiometric composition. After the PZT deposition, top electrode 100-nm-thick Pt was deposited by the dc sputtering method.

For the measurement of the piezoelectric properties of the PZT films, Rectangular beams (cantilevers) with the size of about $30 \mathrm{~mm}(3 \mathrm{~cm}) \times 3 \mathrm{~mm}$ were prepared. Polarization and displacement in these films were simultaneously observed using the laser doppler vibrometer (Graphtec AT-3600) and the laser interferometer (Graphtec AT-0023) which were attached to a ferroelectric test system.

Fig. 22 shows relationship between $\mathrm{Pb}$ composition, deposition rate and repeatability. As a result, stable transition of $\mathrm{Pb}$ content within film in continuous sputtering has been confirmed. As can be seen from the figure, the change in the deposition rate was $2.1 \mu \mathrm{m} \pm 1.4 \%$, and changing $\mathrm{Pb}$ composition was $1.0 \pm 0.1 \%$ in the short running for 35 pieces (total thickness; $105 \mu \mathrm{m})$.

Fig. 23 shows XRD patterns of as-sputtered PZT film. No pyrochlore phase can be confirmed and the film appears to be almost perovskite phase with the preferred orientation to (001) or (100). It confirmed that the uniformity of the crystalline property were good in 6 inch area. In addition, the dielectric constant of these samples was measured as shown in Fig.24. As a result, excellent properties with the dielectric constant over 1000 and its uniformity of $\pm 4.7 \%$ were confirmed. 


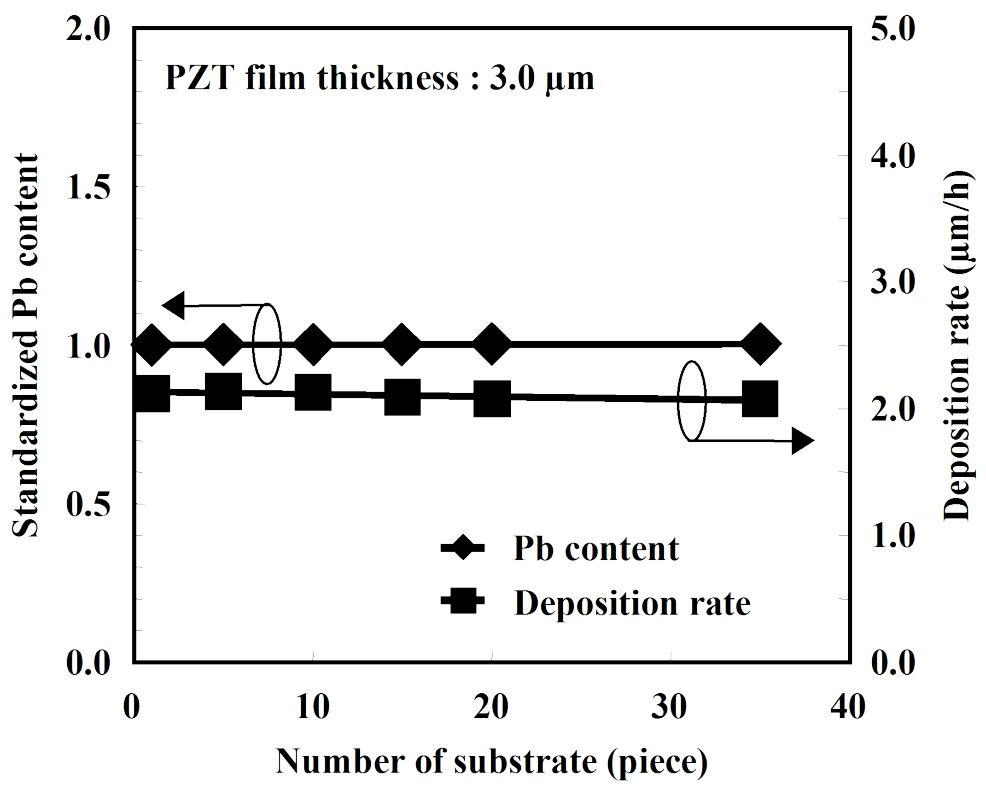

Figure 22. Relationship between $\mathrm{Pb}$ composition, deposition rate and repeatability

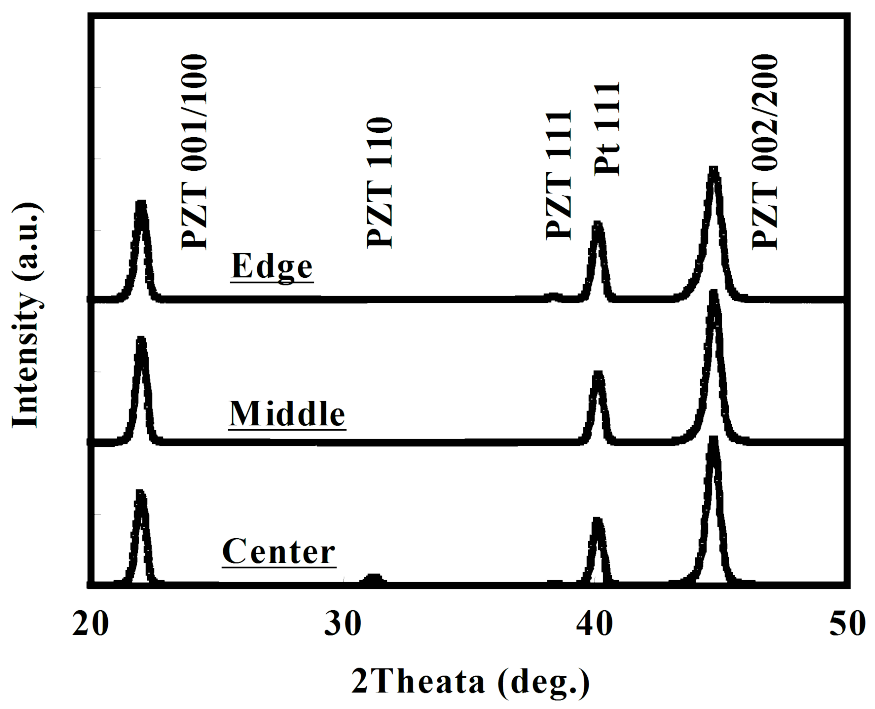

Figure 23. Crystallization uniformity of PZT films Deposited on 6-inches substrate 


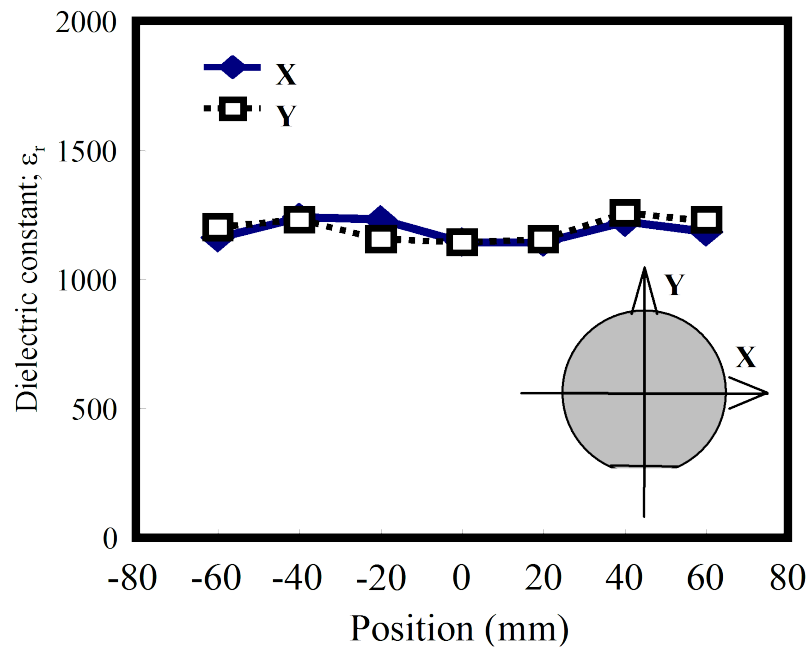

Figure 24. Dielectric constant of PZT films deposited on 6 inch substrate

Piezoelectric properties were finally confirmed for PZT films by checking the cantilever as shown in Fig. 25. Large piezoelectric coefficient from -60 to $-120 \mathrm{pm} / \mathrm{V}$ was observed in our PZT films.

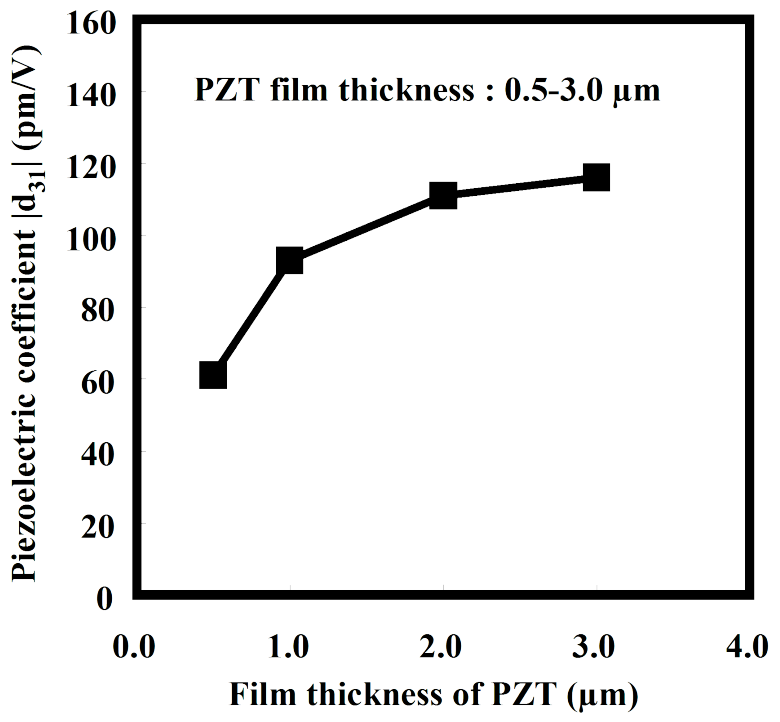

Figure 25. Relationship between piezoelectric coefficient and PZT film thickness 


\subsection{Etching technology of PZT piezoelectric Films for MEMS application}

Additionally etching technology for mass production is developed. Since high etching rate is required in MEMS PZT process because of large PZT thickness, etching selectivity to the bottom electrode $(\mathrm{Pt})$ and uniformity are important. In Fig. 26 we show etching rate profile of both electrode(Pt) and PZT film. It has been found that etching rate of $\mathrm{Pt}$ is smaller than PZT over whole wafers, implying that $\mathrm{Pt}$ film is the role of an etch stop layer.

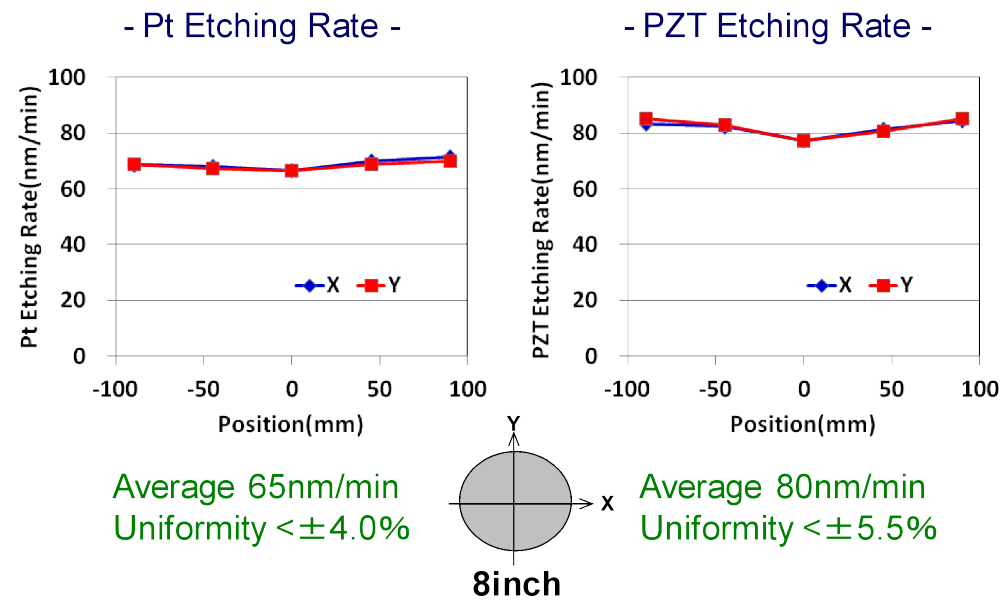

Figure 26. Etching rate and in-plane uniformity of Pt \& PZT

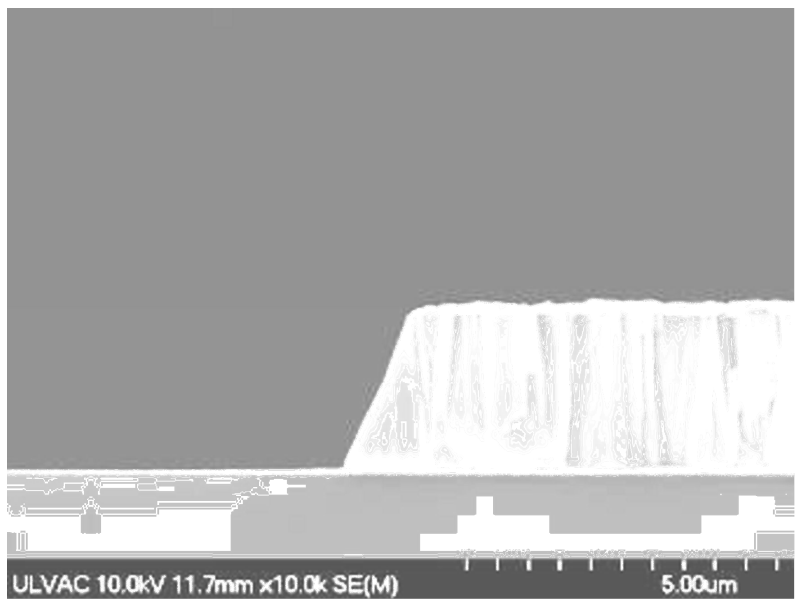

Figure 27. SEM image of Pt/PZT etching profile 
Fig. 27 shows the SEM image after the piezoelectric element was etched and the resist mask was removed. The film composition is $\mathrm{Pt} / \mathrm{PZT} / \mathrm{Pt}=100 \mathrm{~nm} / 3 \mu \mathrm{m} / 100 \mathrm{~nm}$, and the top Pt electrode and PZT were continuously etched by using a $5-\mu \mathrm{m}$-thick photo resist as a mask. Chlorine and fluorine mixed gases are used for PZT etching. The etching shape (taper angle) is about $65^{\circ}$, and nothing adhered to the pattern sidewalls. Despite $20 \%$ of over etching, the bottom Pt electrode was hardly etched. This indicates that a high etching selectivity to $\mathrm{Pt}$ was achieved. Fig. 28 shows the dependency of the etching rate and the taper angle on the bias power. As the bias power increases, the etching rate increases linearly. When $400 \mathrm{~W}$ was applied, the PZT etching rate of $190 \mathrm{~nm} / \mathrm{min}$. was achieved. The taper angle also increased gradually as the bias power increased.

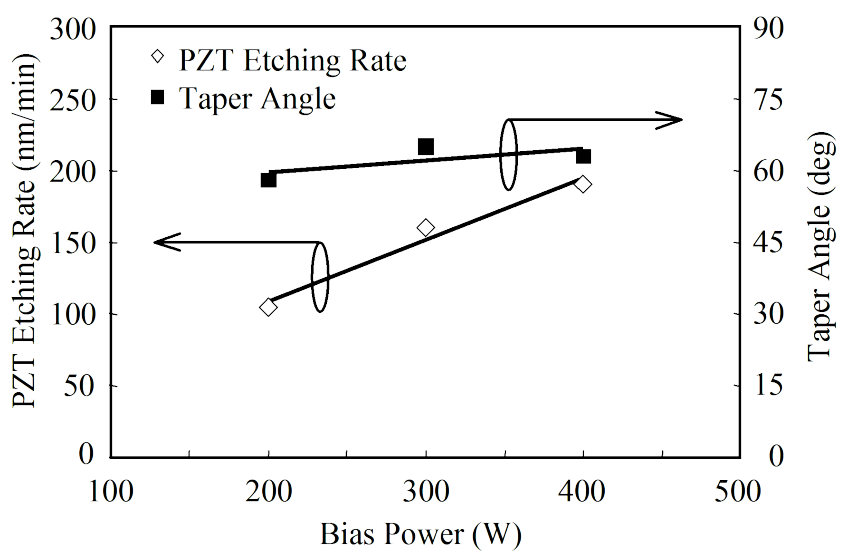

Figure 28. Dependence of PZT etching rate and taper angle on the substrate bias power

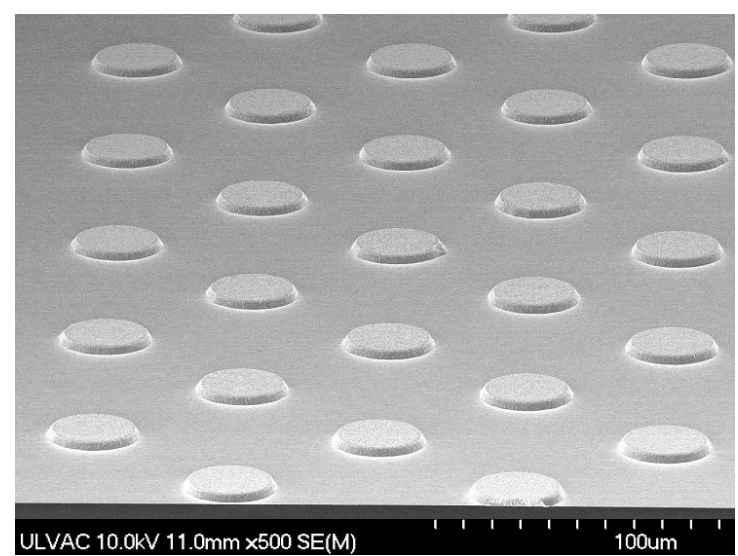

Figure 29. Pt/PZT etching profile of $\$ 50 \mathrm{~mm}$ piezoelectric element array 
Fig. 29 shows a $50-\mu$ m-diameter actuator element array fabricated by dry etching. Fig. 30 shows that the remanent polarization ( $\mathrm{Pr}$ value) of the piezoelectric thin-film actuator with a 3- $\mu$ m-thick PZT film was $40.5-42.8 \mu \mathrm{C} / \mathrm{cm}^{2}$ and the coercive electric field ( $\mathrm{E}_{\mathrm{c}}$ value) was $44.5-46.0 \mathrm{kV} / \mathrm{cm}$ at an applied voltage of $30 \mathrm{~V}$, and the characteristics without the dependency on element size (30-300 $\mu \mathrm{m}$ diameter) were obtained. The damage to the PZT piezoelectric thin-film actuator caused by the dry etching is considered to be negligible. The displacement of the PZT thin-film actuator was measured by contact-AFM. Fig.31 shows that a displacement of about $4 \mathrm{~nm}$ was obtained at 3- $\mu$ m-thick PZT film, 30- $\mu \mathrm{m}$-diameter element size, and an applied electric field of $100 \mathrm{kV} / \mathrm{cm}$. It was clarified that the processing of PZT piezoelectric thin-film actuators by dry etching is very effective.

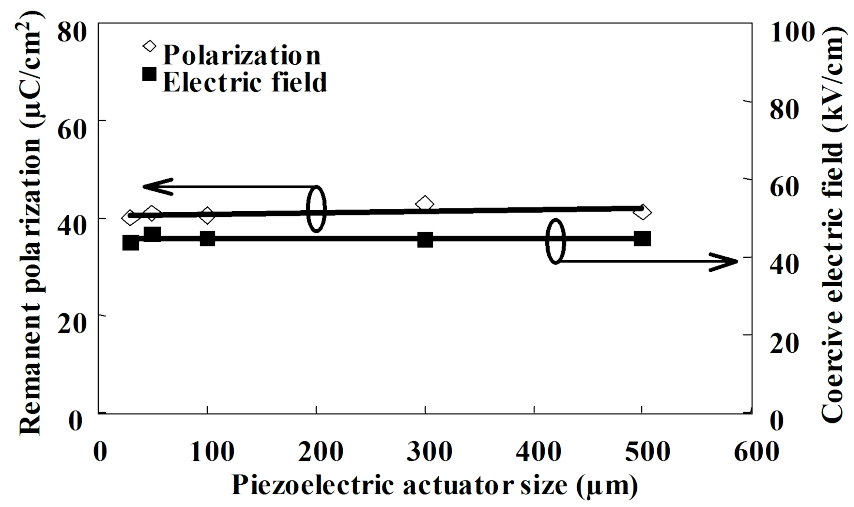

Figure 30. Dependence of ferroelectric properties on piezoelectric actuator size

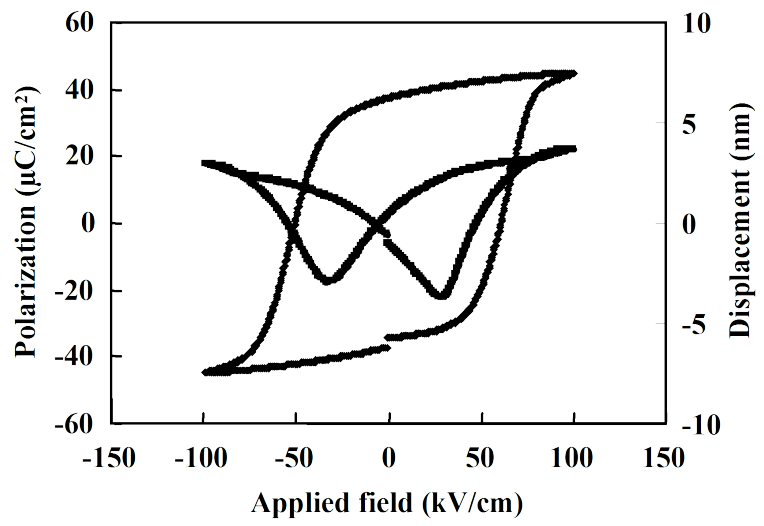

Figure 31. Ferroelectric and displacement properties of Pt/PZT/Pt element with $30 \mathrm{~mm}$ diameter etching rate and taper angle on the substrate bias power 


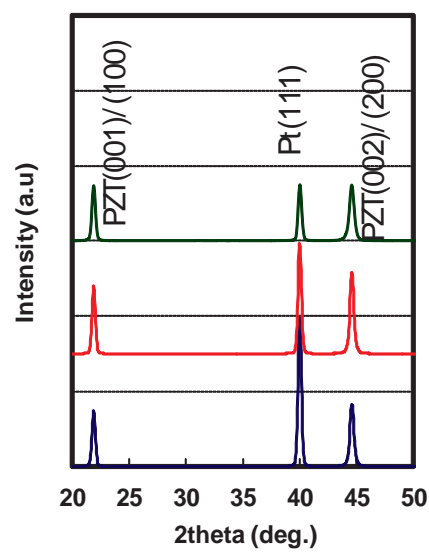

(Conventional process)

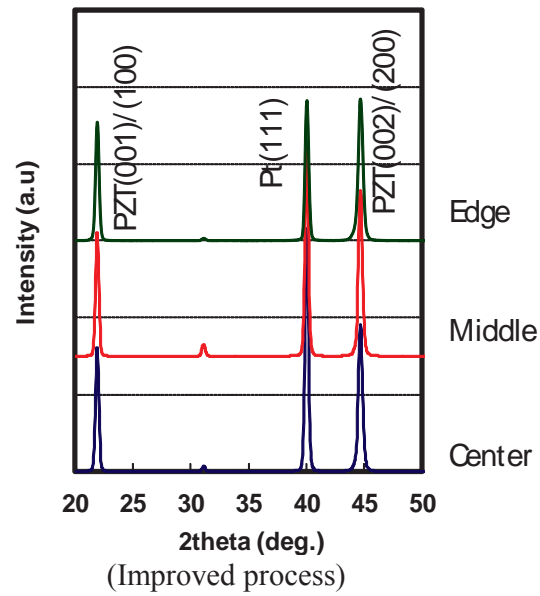

(Improved process)

Figure 32. XRD Patterns of PZT film before and after improvement on 8inch $\mathrm{Pt} / \mathrm{Ti} / \mathrm{SiO}_{2} / \mathrm{Si}$ Substrate

\subsection{Further development for MEMS application}

We also note that post in-situ treatment [17] has been recently established, aiming to improve crystalline property and piezoelectric coefficient in our PZT films. Fig. 32 shows the results before and after improvement of crystalline properties. Peak intensity of a/c axis of PZT film deposited by improved process is approximately two times higher of peak intensity of PZT film deposited by conventional process uniformity over 8 inch wafers. As a consequence good piezoelectric coefficient $\left(12.9 \mathrm{C} / \mathrm{m}^{2}\right)$ and large breakdown voltage $(68 \mathrm{~V})$ were obtained for PZT film deposited by improved sputtering process in Fig 33.

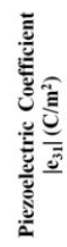

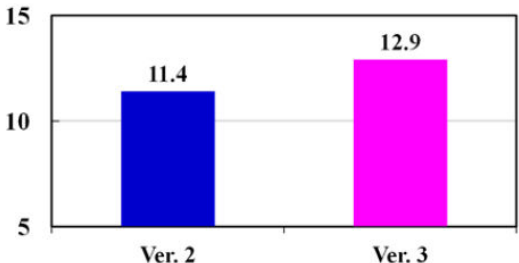

Conventional Improved

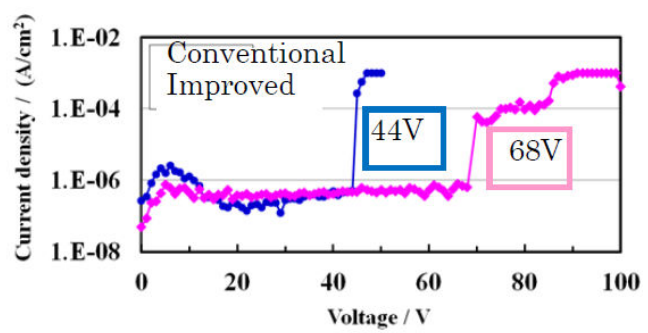

Figure 33. Improvement of piezoelectric coefficient(Left) and break down voltage(Right) 
Good piezoelectric performance which is based on mass production technology including excellent in-plane uniformity and process rate of sputtering and etching was obtained in above evaluation.

\subsection{Sputtered BST thin-films for capacitor and RF tunable devices}

$(\mathrm{Ba}, \mathrm{Sr}) \mathrm{TiO}_{3}$ (BST) is expected to use as the thin film capacitor, $\mathrm{RF}$ tunable component $[18,19]$ on-chip capacitor [20] for its excellent dielectric behavior. In these applications, decoupling, high permittivity and high tunability are required as dielectric characteristics. High permittivity is thought to be primarily important character as dielectric characteristics. BST thin films have been deposited by some deposition techniques. Among these techniques, RF magnetron sputtering is thought to be suitable for mass-production because of its stability and reproducibility as well as good film performances. In this section, BST films deposition by RF magnetron sputtering and an approach to deposit BST films with higher permittivity were described.

BST films were also deposited by an RF magnetron sputtering method using sintered BST ceramic targets. Basic sputtering conditions such as RF power, deposition temperature and so on were varied. As a top electrode, Pt dot with $0.5 \mathrm{~mm}$ in diameter was deposited by DC magnetron sputtering. After top electrode deposition, BST capacitors were post-annealed for 1 hour in oxygen atmosphere under 1 atm using resistive heat furnace.

Fundamental properties of BST thin films such as dielectric constant, crystalline quality (XRD), surface morphology (SEM) were investigated. Agilent 4284A LCR meter was used for dielectric properties measurement and measuring cycle and volts alternating current were $1 \mathrm{kHz}$ and $1 \mathrm{Vrms}$, respectively.

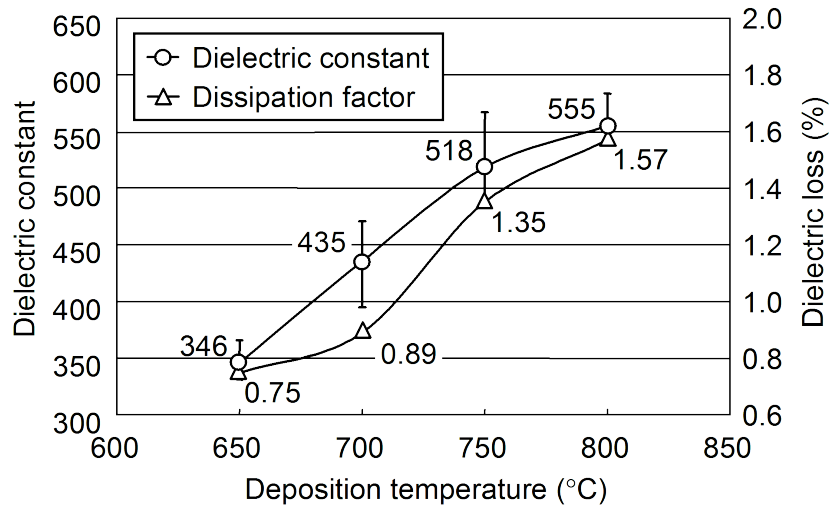

Figure 34. Relationship between dielectric constant and deposition temperature

The relationship between dielectric constant and deposition temperature was investigated as shown in Fig. 34. Ba/Sr ratio of BST target was 50/50. RF power was $1500 \mathrm{~W}$. Ar/O $/ \mathrm{O}_{2}$ flow 
ratio was 3. Sputtering pressure was 2.0 Pa. BST film thickness was $100 \mathrm{~nm}$. As a result, deposition rate was $4.8 \mathrm{~nm} / \mathrm{min}$ and almost constant irrespective of deposition temperature. As can be seen in this figure, dielectric constant is strongly dependent on deposition temperature and increasing with deposition temperature increasing. XRD patterns of these films are also shown in Fig. 35. We can see in this figure, BST grains are randomly orientated and XRD peak intensities from BST films are increasing with deposition temperature increasing. So, it is thought that this represents the relationship between dielectric constant and deposition temperature.

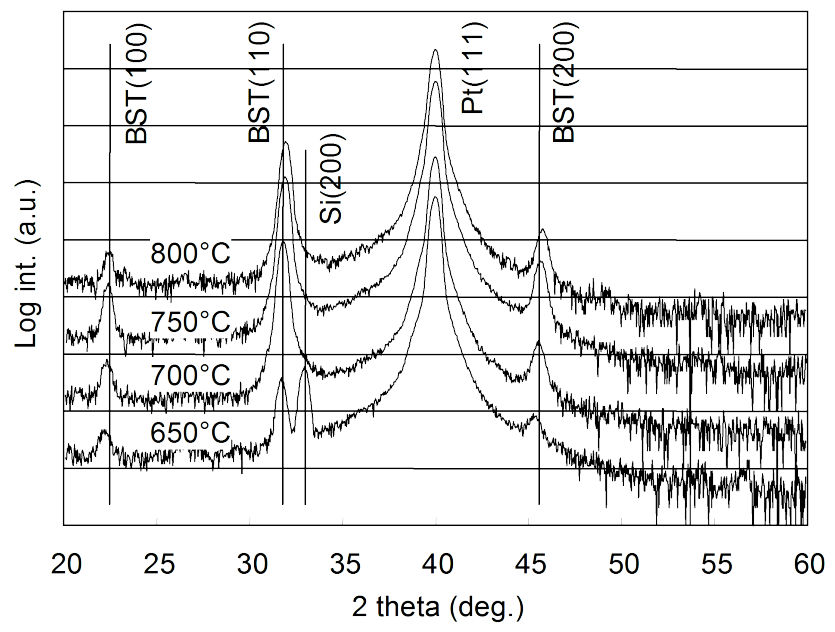

Figure 35. Relationship between XRD pattern and deposition temperature

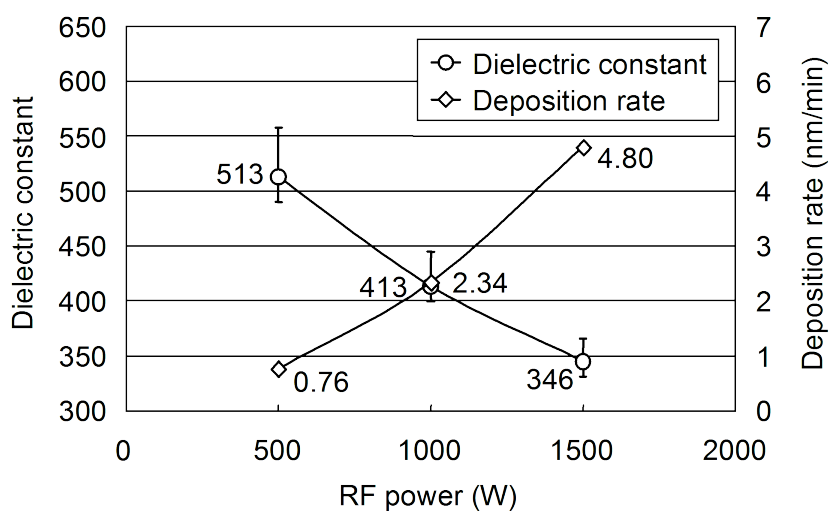

Figure 36. Relationship between dielectric constant, deposition rate and RF power temperature 
The relationship between dielectric constant, deposition rate and RF power is shown in Fig. 36. Deposition temperature was $650^{\circ} \mathrm{C}$ in this relationship, while other conditions were not changed. We can see in this figure, there is trade-off relationship between dielectric constant and deposition rate and higher dielectric constant can be obtained at low sputtering power of $500 \mathrm{~W}$ in this experiment. BST morphology (SEM photographs) dependence on RF power is also shown in Fig. 37. As can be seen in this figure, BST film deposited at $500 \mathrm{~W}$ (b) has dense and uniform columnar structure. Possibly it represents some relationship between dielectric constant and RF power. It is speculated that there is a long time for atomic migration and the growth of BST crystal is encouraged under such low deposition rate around $1 \mathrm{~nm} / \mathrm{min}$.
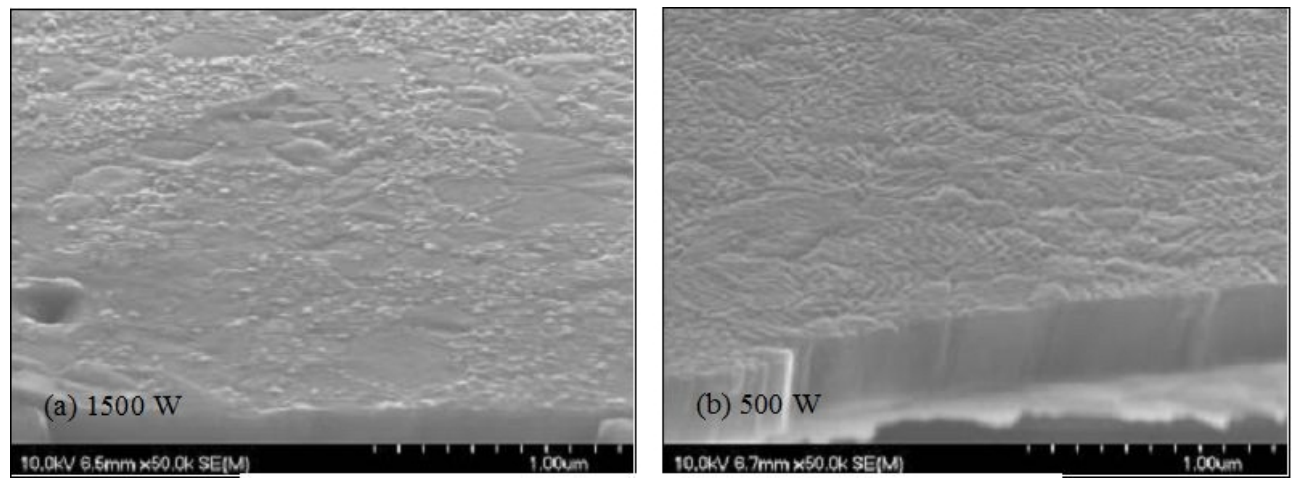

Figure 37. BST morphology dependence on RF power

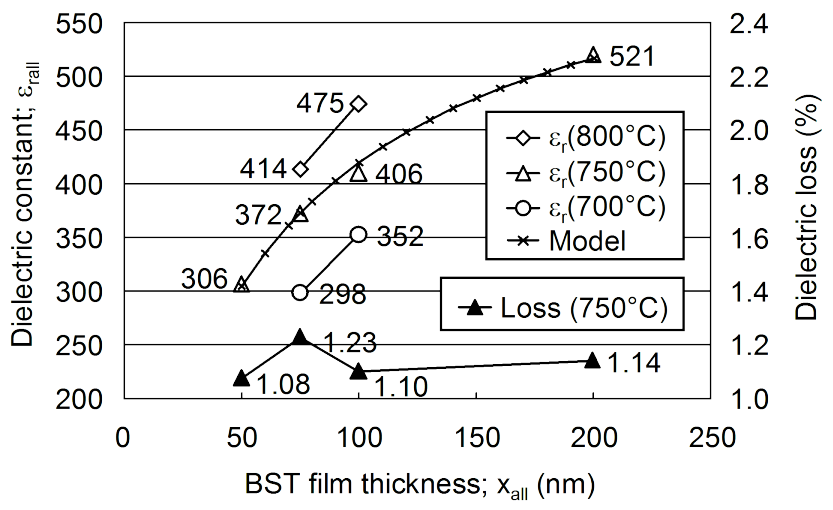

Figure 38. Relationship between dielectric constant and BST film thickness

Furthermore, the relationship between dielectric constant and BST film thickness is shown in Fig. 38. Ba/Sr ratio of BST target was 70/30 in this experiment. Deposition temperature was varied from $700^{\circ} \mathrm{C}$ to $800^{\circ} \mathrm{C}$ as a parameter, while other conditions were not changed. 
We can see in this figure, dielectric constant is also strongly and non-linearly dependent on BST film thickness.

If BST capacitor is assumed to be a simple series connected capacitor between transition layer near BST/Pt bottom electrode interface and main layer which represents BST film excluding transition layer, it is speculated that there have been the transition layer in this BST film. [21]

As a simple experiment, effects of gas flow sequence for BST deposition was investigated. Gas flow sequence, that is ON/OFF step was changed, while $\mathrm{Ar} / \mathrm{O}_{2}$ flow ratio was not changed. Dielectric constant was improved by introducing of oxygen gas before BST deposition. It is speculated in this experiment that BST/Pt interface was improved because the oxygen vacancies of BST in this region were reduced. Therefore, dielectric properties are noticeably influenced by gas flow sequencing variation.

\section{Technology for ferroelectric probe memory}

Technology for ferroelectric probe memory was developed with the deposition technology for FeRAM manufacturing. Hard-disk using the magnetic recording is the one of the major storage device, but the recording density will reach the limit in the near future by exteriorization of the magnetization disappearance by the heat disturbance. Ferroelectric probe memory is ultrahigh-density memory applied Scanning Probe Microscope (SPM) and ferroelectric property.

Ferroelectric perovskite $\mathrm{Pb}(\mathrm{Zr}, \mathrm{Ti}) \mathrm{O}_{3}(\mathrm{PZT})$ is polarized by electric field. For scanning probe memory device, ferroelectric layer required atomically-smooth surface and low leakage current to achieve large recording density by forming polarized domains as unit cell size. Therefore it is necessary to grow the epitaxial ferroelectric thin films oriented to $c$-axis direction.

In this time we prepared epitaxial growth $\mathrm{PZT} / \mathrm{SrRuO}_{3}(\mathrm{SRO})$ thin films on single crystal $\mathrm{SrTiO}_{3}(\mathrm{STO})$ substrate for probe memory device, and evaluated the film properties and the recording density with Piezoresponse force microscopy (PFM). The SRO films were deposited on STO (100) single crystal substrate with DC sputtering. After that, the PZT film was deposited by MOCVD process.

Fig. 39 shows the AFM image of PZT / SRO / STO deposited at the optimal Pb flux. The surface morphology of PZT has step structure and smooth terraces similar to SRO/STO substrate before PZT deposition. This surface profile indicates layer-by-layer growth of PZT on SRO / STO substrate [22] [23] [24]. Fig. 40 shows XRD spectrum of PZT deposited at Pb flux of $0.160 \mathrm{ml} / \mathrm{min}$. There are only PZT, SRO and STO peaks. The fringe pattern appears around PZT (001) and (002) peaks because of the smooth interface between PZT and SRO. Fullwidth at half-maximum (FWHM) of PZT (002) with X-ray rocking curve is 0.129degree for optimal $\mathrm{Pb}$ flux of $0.160 \mathrm{ml} / \mathrm{min}$. Rms of surface roughness and PZT(002) FWHM of X-ray rocking curve have a similar trend relative to $\mathrm{Pb}$ flux. 


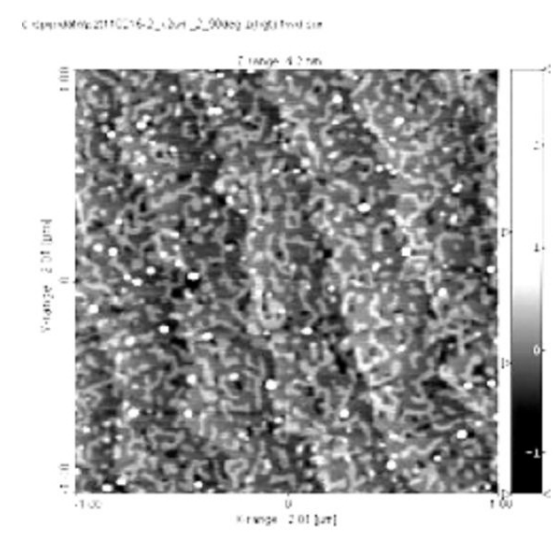

Figure 39. AFM image of $\mathrm{PZT}$ at $\mathrm{Pb}$ flux of $0.160 \mathrm{ml} / \mathrm{min}$ (Scanning range is 2 um square)

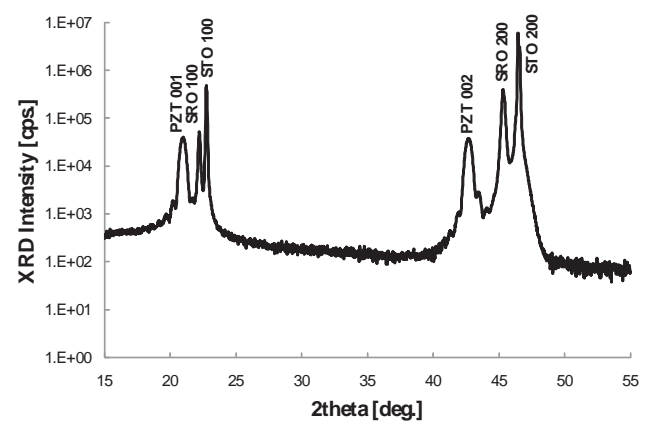

Figure 40. XRD spectrum of PZT / SRO /STO

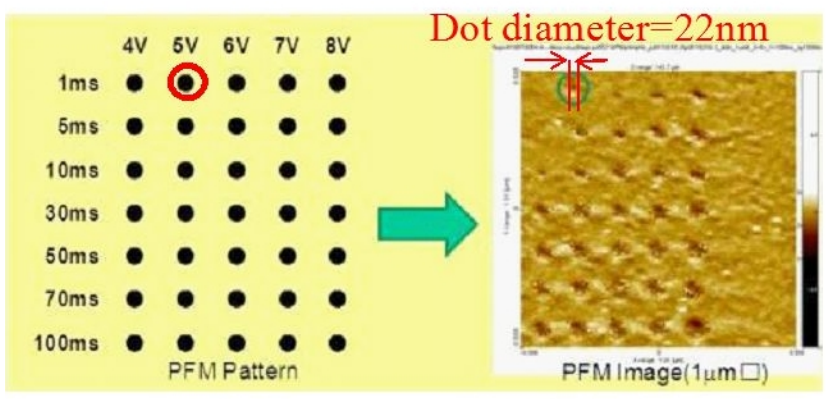

Figure 41. Writing Voltage \& Writing Time Dependency of Dot diameter - [Small diameter (22nm) of recording-dot was obtained for 5V \& $1 \mathrm{~ms}$ writing condition. (PZT/SRO/c-STO) • Estimated Recording density: 1.4Tbit/inch². ] 
PFM measurement is carried out with various biases between $4 \mathrm{~V}$ and $8 \mathrm{~V}$ and pulse widths between $1 \mathrm{msec}$ and $100 \mathrm{msec}$ with PtIr $_{5}$ coatedprobe (tip diameter is $25 \mathrm{~nm}$ ). Fig. 41 shows PFM write-condition and PFM image of the result. Polarization-inverted dot diameter has a minimum of $20 \mathrm{~nm}$ at the bias of $5 \mathrm{~V}$ and pulse width of $1 \mathrm{msec}$. Recording density is estimated on the assumption that this tiny dot is arrayed with pitch of $20 \mathrm{~nm}$. From the calculation result, device sample deposited under the optimal growth condition achieves large bit density of $1.4 \mathrm{~Tb} /$ inch $^{2}$.

\section{Conclusion}

We have been developing thin film process technologies for ferroelectric application of advanced semiconductor and electronics usage for 20 years or more, and completed the ferroelectric thin film solutions (sputtering, MOCVD, and etching) that became a de facto standard. These technologies will support a wide variety of convenient energy-saving devices such as FeRAM, MEMS production (actuators composing gyro meters, portable camera modules for smart phone applications, tunable devices and so on), and ultra-high density probe memory.

\section{Author details}

Koukou Suu

Institute of Semiconductor and Electronics Technologies, ULVAC, Inc., Shizuoka, Japan

\section{References}

[1] K. Suu, A. Osawa, N. Tani, M. Ishikawa, K. Nakamura, T. Ozawa, K. Sameshima, A. Kamisawa and H. Takasu: Jpn. J. Appl. Phys. 35 (1996) 4967.

[2] K. Suu, A. Osawa, N. Tani, M. Ishikawa, K. Nakamura, T. Ozawa, K. Sameshima, A. Kamisawa and H. Takasu: Integr. Ferroelectr. 14 (1997) 59.

[3] K. Suu, A. Osawa, Y. Nishioka and N. Tani: Jpn. J. Appl. Phys. 36 (1997) 5789.

[4] K. Suu, Y. Nishioka, A. Osawa and N. Tani: Oyo Buturi 65 (1996) 1248. (in Japanese)

[5] K. Suu: Proc. Semicon Korea Tech. Symp., 1998 p. 255.

[6] N. Inoue, Y. Maejima and Y. Hayashi: Int. Electron Device Meet. Tech. Dig., 1997 p. 605.

[7] N. Inoue, T. Takeuchi and Y. Hayashi: Int. Electron Device Meet. Tech. Dig., 1998 p. 819. 
[8] F. Chu, G. Fox, T. Davenport, Y. Miyaguchi and K. Suu: Integr. Ferroelectr. 48 (2002) 161.

[9] T. Yamada, T. Masuda, M. Kajinuma, H. Uchida, M. Uematsu, K. Suu and M. Ishikawa : IFFF2002 abstract, 4 (2002) 37.

[10] T. Masuda, M. Kajinuma, T. Yamada, H. Uchida, M.Uematsu, K. Suu and M. Ishikawa : Integr. Ferroelectr., 46 (2002) 66

[11] Y. Nishioka, T. Jinbo, T. Yamada, T. Masuda, M. Kajinuma, M. Uematsu, K. Suu and M. Ishikawa: Integr. Ferroelectr., 59 (2003) 1445

[12] Y. Nishioka, T. Masuda, M. Kajinuma, T. Yamada, M. Uematsu and K. Suu: MRS Fall Meeting Proceedings, 784-C7 (2003) 6

[13] K. Zheng, J. Lu, and J. Chu: Jpn. J. Appl. Phys. 43 (2004) 3934.

[14] Y. Kokaze, M. Endo, M. Ueda, and K. Suu: 17th Int. Symp. Integrated Ferroelectrics, 2005, 5-26-P.

[15] Y. Kokaze, I Kimura, M. Endo, M. Ueda, S. Kikuchi, Y. Nishioka, and K. Suu: Jpn. J. Appl. Phys. 46 (2007) 282.

[16] M. Endo, M. Ueda, Y. Kokaze, M. Ozawa, T. Nakamura, K. Suu: SEMI Technology Symposium 2002; December 5, 2002

[17] Suu et.al in preparation

[18] I. P. Koutsaroff, T. Bernacki, M. Zelner, A. Cervin-Layry, A. Kassam, P. Woo, L. Woodward, A. Patel, Mat. Res. Soc. Symp. Proc. Vol. 762, C5.8.1 (2003).

[19] X. H. Zhu, J. M. Zhu, S. H. Zhou, Z. G. Liu, N. B. Ming, S. G. Lu, H. L. W. Chen, C. L. Choy, Journal of Electronic Materials, 32 (2003) 1125.

[20] M. C. Werner, I. Banerjee, P. C. McIntyre, N. Tani, M. Tanimura, Appl. Phys. Lett., 77 (2000) 1209.

[21] T. Jimbo, I. Kimura, Y. Nishioka and K. Suu, Mat. Res. Soc. Symp. Proc. Vol. 784, C7.8.1 (2003).

[22] P.-E. Janolin, B. Fraisse, F. Le Marrec, and B. Dkhil, Appl. Phys. Lett. 90, 212904 (2007)

[23] Keisuke Saito, Toshiyuki Kurosawa, Takao Akai, Shintaro Yokoyama, Hitoshi Morioka, Takahiro Oikawa, and Hiroshi Funakubo, Mater. Res. Soc. Symp. Proc. 748, U13.4.1-U13.4.6 (2003).

[24] H. Hu, C. J. Peng and S. B. Krupanidhi, Thin Solid Films, 223 (1993) 327 333I. G. Baek, et al,: Tech. Dig. Int. Electron Devices Meet., San Francisco, 2004, 23, 6, p58 
